\title{
Multicomponent synthesis and anticonvulsant activity of monocyclic 2,6-diketopiperazine derivatives
}

\author{
Maciej Dawidowski · Jadwiga Turło
}

Received: 8 May 2013/Accepted: 19 September 2013/Published online: 1 October 2013

(c) The Author(s) 2013. This article is published with open access at Springerlink.com

\begin{abstract}
In this study, a series of diastereomerically pure monocyclic 2,6-diketopiperazine (2,6-DKP) derivatives were synthesized. The key synthetic step involved a multicomponent Ugi five-center, four-component reaction which was used to generate the convertible tert-butylamidoesters with both good yields and high diastereoselectivity toward the desired bioactive $(S, S)$ absolute configuration. In subsequent steps, selective tertbutyl cleavage by use of $\mathrm{BF}_{3} \cdot \mathrm{CH}_{3} \mathrm{COOH}$ and base-induced intramolecular cyclocondensation gave the final 2,6-DKP derivatives. The relative stereochemistry of the target molecules was confirmed by ${ }^{1} \mathrm{H}$ NMR experiments. The compounds obtained were submitted to in vivo screening in animal models of epilepsy. Some of them displayed good activity in maximal electroshock seizure and $6 \mathrm{~Hz}$ tests.
\end{abstract}

Keywords Multicomponent reactions - Ugi reaction Anticonvulsant activity · 2,6-Diketopiperazine ·

2,6-Piperazinedione

\section{Introduction}

Epilepsy is a major neurological disorder characterized by recurrent, spontaneous seizures. It affects approx. 50 million people ( $\sim 1 \%$ of the world's population). Currently, the main treatment for epilepsy is the chronic administration of anticonvulsant drugs (AEDs). Although more than 30 AEDs are available, they provide satisfactory seizure

\section{Dawidowski $(\bowtie) \cdot J$. Turło}

Department of Drug Technology and Pharmaceutical

Biotechnology, Medical University of Warsaw, Banacha 1 Str., 02-097 Warsaw, Poland

e-mail: maciej.dawidowski@wum.edu.pl control in only $60 \%$ of patients. Additionally, major concerns of pharmacotherapy of epilepsy include high incidence of severe side effects and drug-drug interactions resulting from enzyme induction. Therefore, there is substantial need for new, more effective and safer AEDs (Kwan and Brodie, 2000; Brodie, 2001). Another essential challenge for epilepsy research is to develop therapeutics that would not only symptomatically suppress seizures, but would also inhibit or reverse progression of the sickness (the so-called "disease modifying" drugs; Perucca et al., 2007; Bialer and White, 2010).

Presently, the compounds at different stages of development belong to various chemical classes and display diverse, often unknown mechanisms of action (Bialer et al., 2013). Most of these agents have been identified initially through in vivo screening in animal models of epilepsy rather than by a mechanistic approach. Although the animal models utilized for screening are associated with certain endpoints, it is generally accepted that they offer a good starting point in the early discovery of new AED candidates (Löscher and Schmidt, 1994; Malawska, 2005; Rogawski, 2006; Smith et al., 2007; Bialer and White, 2010; Banerjee and Sharma, 2012; Mishra and Ganguly, 2012).

Recently, we have reported that chiral, bicyclic 2,6diketopiperazines (2,6-DKPs) derived from cyclic amino acids display a broad anticonvulsant activity in various animal models of epilepsy (Dawidowski et al., 2011, 2012a). Among the newly developed agents, compound ADD408003 exhibited a broad spectrum of seizure-suppressing activity. A preliminary structure-activity relationship (SAR) study of close analogs revealed that several factors are responsible for the anticonvulsant activity (Fig. 1): the $(S, S)$ absolute configuration on the stereogenic centers, the presence of imide moiety and the benzene ring adjacent to 2,6-DKP core. Further, neither substitution of 
the imide nitrogen of ADD408003 with different alkyl and arylalkyl moieties nor expansion of the fused pyrrole chain markedly influenced the antiseizure activity.

These findings led us to ask whether the related monocyclic 2,6-DKPs, derived from non-polar L-amino acids other than L-proline or L-homoproline display comparable anticonvulsant activity. The designed compounds fulfill all requirements determined on the basis of the preliminary SAR analysis, i.e., proper stereochemistry, the presence of imide moiety and benzene ring attached to 2,6-DKP scaffold. Further, due to the absence of the fused pyrrolidine or piperidine rings, these agents are less sterically constrained, which might allow for a better fit to the putative receptor(s).

\section{Results and discussion}

\section{Chemistry}

The target enantiopure, monocyclic 2,6-DKP derivatives 3a-e were synthesized according to the reaction sequences depicted in Scheme 1.

In the first step, the Ugi five-center four-component reaction (U-5C-4CR; Demharter et al., 1996) of the appropriate non-polar amino acid (L-valine, L-leucine, L-isoleucine, L-phenylalanine, L-phenylglycine), benzaldehyde, tert-butyl isocyanide, and methanol in the presence of a catalytic amount of iron(III) chloride gave the tertbutylamidoesters 1a-e with chemical yields ranging from

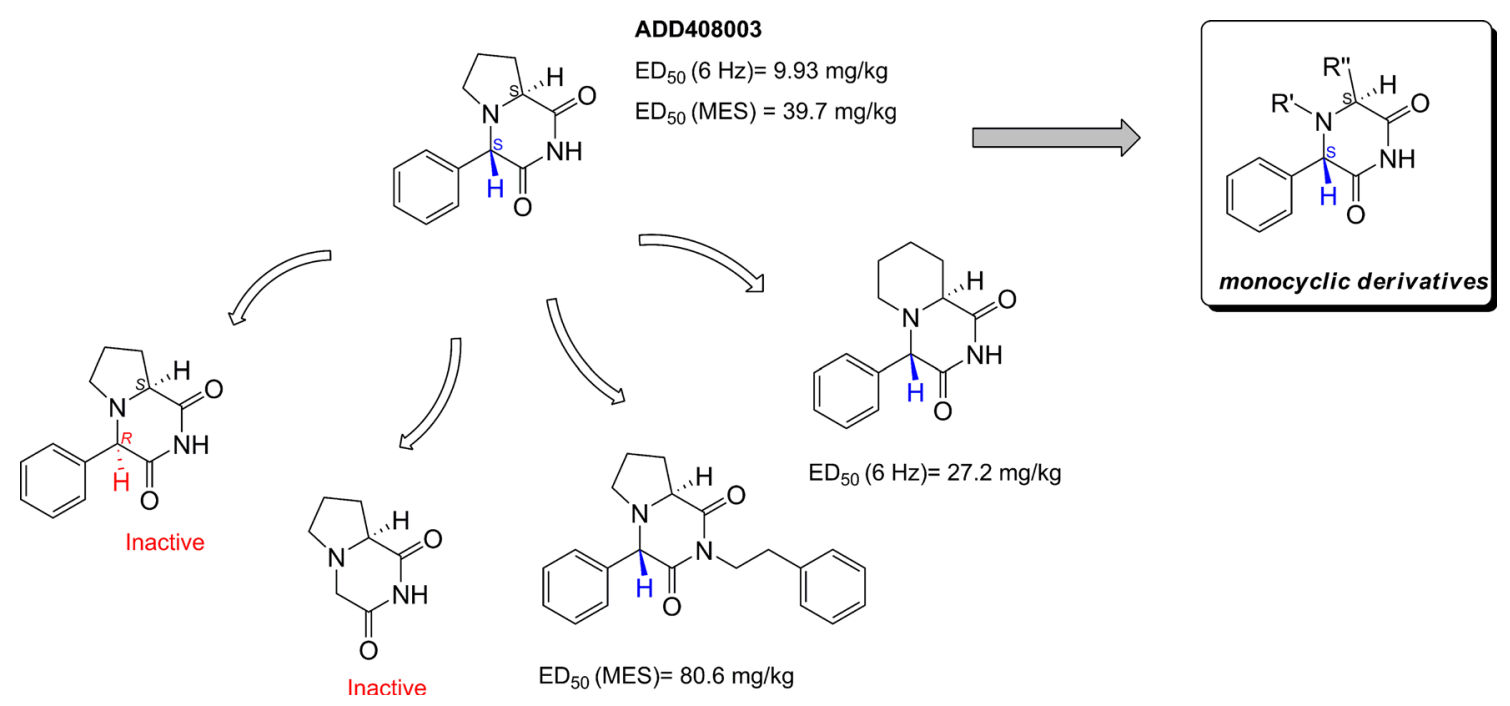

Fig. 1 Preliminary SAR of anticonvulsant 2,6-DKPs and proposed structural modifications<smiles>[R]C(N)C(=O)OCC(=O)NC(NC(C)(C)C)C(=O)NC([R])C(C)C</smiles><smiles>[R]C(NC(C(N)=O)c1ccccc1)C(=O)OC</smiles><smiles>[R]C1NC(c2ccccc2)C(=O)NC1=O</smiles><smiles>[R]C1NC(=O)C(=O)NC1=O</smiles>

$(2 S, 1 R)$ - and $(2 S, 1 S)-1 \mathrm{a}, \mathrm{b}, \mathrm{c}, \mathrm{d}, \mathrm{e}$

$(2 S, 1 S)-2 a, b, c, d$

$(3 S, 5 S)-3 a, b, c, d, e$

$(3 R, 5 S)-3 \mathrm{a}, \mathrm{b}, \mathrm{c}, \mathrm{d}, \mathrm{e}$

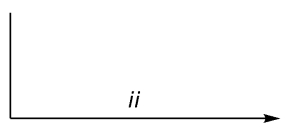<smiles>COC(=O)C(Nc1ccccc1)c1ccccc1</smiles><smiles>CC#CC</smiles>

$(2 S, 1 R)-$ and $(2 S, 1 S)-2 \mathrm{e}$

i. $\mathrm{PhCHO}, t \mathrm{BuNC}, \mathrm{MeOH}, \mathrm{FeCl}_{3}$ (cat.), rt, $48 \mathrm{~h}$

ii. $\mathrm{BF}_{3}{ }^{*} 2 \mathrm{CH}_{3} \mathrm{COOH}, 45-55{ }^{\circ} \mathrm{C}, 4-6 \mathrm{~h}$

iii. chromatographic resolution of diastereoisomers iv. $\mathrm{NaOH}, \mathrm{EtOH}, \mathrm{tr}, 10$ min.<smiles>[R]=[SH]CC(C)C(C)CC(C)CC(C)CC(C)(C)[C@H](C)CC</smiles>

Scheme 1 Synthesis of enantiopure 2,6-DKP derivatives 3a-e 
41 to $75 \%$. The reaction proceeded with the formation of the new stereocenter and in all cases, the major diastereomer was $(\mathbf{2 S}, \mathbf{1 S})-\mathbf{1}$, as judged by the ${ }^{1} \mathrm{H}$ NMR analyses of the crude post-reaction mixtures. In general, the degree of diastereoinduction depended on the steric bulkiness of the side chain of the substrate amino acid. The highest diastereomeric ratios were measured for L-valine and L-isoleucine derivatives 1a $\left(d_{\mathrm{r}}=7.3 / 1\right)$ and 1c $\left(d_{\mathrm{r}}=9.0 / 1\right)$, respectively, bearing branched alkyl chains directly adjacent to the position $\mathrm{C}-2$, located close to the newly formed stereocenter. The U-5C-4CR adducts of L-leucine and L-phenylalanine $\mathbf{1 b}$ and $\mathbf{d}$, respectively, were formed with a slightly lower diastereoinduction $\left(d_{\mathrm{r}} \approx 5 / 1\right.$ for each). This could be attributed to the lower steric hindrance of a methylene group adjacent to the carbon $\mathrm{C}-2$. A surprisingly small degree of diastereoselectivity was found for the L-phenylglycine derivative 1e $\left(d_{\mathrm{r}}=1.5 / 1\right)$, having a bulky phenyl substituent in the position $\mathrm{C}-2$. The possible explanation for this unexpected observation is the stabilization of the six-membered cyclic Ugi intermediate (Demharter et al., 1996) leading to $(\mathbf{2 S , 1 R})-\mathbf{1 e}$ by a pi-pi interaction of the two phenyl rings occupying axial positions.

Attempts to quantitatively separate the diastereoisomers of 1a-e by column chromatography or fractional recrystallization failed. Therefore, the obtained diastereomeric mixtures were used in the subsequent amide $\mathrm{N}$-detertbutylation. Reaction of $(\mathbf{2 S , 1 S ) / ( 2 S , 1 R ) - 1 a - e ~ w i t h ~}$ $\mathrm{BF}_{3} \cdot \mathrm{CH}_{3} \mathrm{COOH}$ at $45-55^{\circ} \mathrm{C}$ provided amidoesters $2 \mathbf{a}-\mathbf{e}$ with the yields range from 55 to $83 \%$. With the exception of $\mathbf{2 e}$, all diastereomeric mixtures could be efficiently resolved by column chromatography.

In the last step, compounds $(\mathbf{2} S, \mathbf{1 S})-\mathbf{2 a}-\mathbf{d}$ were subjected to base-induced intramolecular cyclization. The reaction was accompanied by a notable degree of epimerization at stereogenic centers C-5 of the products 3 . Nevertheless, in all cases, the unwanted $(\mathbf{3 S}, \mathbf{5} \boldsymbol{R})$ isomers could be separated by means of column chromatography (compounds $(\mathbf{3 S}, \mathbf{5} \boldsymbol{R})$ 3a, c, d) or recrystallization (compound (3S,5R)-3b). Intramolecular cyclization of $1.4 / 1$ diastereomeric mixture of $(2 S, 1 S) /(2 S, 1 R)-2 \mathrm{e}$ gave $(3 S, 5 S)-3 \mathrm{e}$ and $(3 S, 5 R)-3 \mathrm{e}$ (a meso compound) in equal proportion. The isomers were efficiently separated by column chromatography.

Relative stereochemistry of the respective diastereoisomers of 2,6-DKPs 3 was confirmed with nuclear Overhauser effect (nOe) ${ }^{1} \mathrm{H}$ NMR experiments (Fig. 2) performed for $(\mathbf{3 S}, \mathbf{5} S)$ and $(\mathbf{3 S}, \mathbf{5 R})-\mathbf{3 a}$. Contrary to $(\mathbf{3 S}, \mathbf{5} R)-3 \mathrm{a}$, the interatomic distance between protons $\mathrm{H}-3$ and $\mathrm{H}-5$ in $(\mathbf{3 S}, \mathbf{5 S})$-3a should exclude any considerable nOe effect. Indeed, the irradiation of the $\mathrm{H}-3$ resonance in $(\mathbf{3 S}, \mathbf{5} R)-\mathbf{3 a}$ resulted in a remarkable enhancement of the H-5 signal $(4.2 \%)$, whereas no increase in intensity for the proton $\mathrm{H}-5$ was observed in the analogous experiment performed for $(\mathbf{3 S}, \mathbf{5 S})-\mathbf{3 a}$. The reverse order of irradiation, consisting in saturation of $\mathrm{H}-5$, led to the enhancement of $\mathrm{H}-3$ resonance in $\mathbf{( 3 S , 5 R ) - 3 a}(4.0 \%)$, but not in $(\mathbf{3 S}, \mathbf{5 S})-\mathbf{3 a}$.

The difference in the chemical shifts of the protons $\mathrm{H}-3$ and $\mathrm{H}-5$ adjacent to the stereogenic carbon atoms was another ${ }^{1} \mathrm{H}$ NMR spectroscopic feature useful for distinguishing between the respective diastereoisomers of $\mathbf{3}$. With the exception of $\mathbf{3 e}$, the signals corresponding to $\mathrm{H}-5$ in $\mathbf{( 3 S , 5 S ) - 3}$ were shifted downfield $(\Delta \delta=0.26-0.38 \mathrm{ppm}$ [parts per million]) compared to those of $(\mathbf{3 S , 5 R})-\mathbf{3}$. This observation was in agreement with the pseudoequatorial arrangement of these protons with respect to the 2,6-DKP ring in $(\mathbf{3 S}, \mathbf{5} S)-3$, and their pseudoaxial position in (3S,5R)-3. On the contrary, resonances of the $\mathrm{H}-3$ protons in all $(\mathbf{3} S, \mathbf{5} S)-3$ isomers were shifted upfield $(\Delta \delta=0.14$ $0.32 \mathrm{ppm})$. Although these protons in both respective diastereoisomers occupy the same pseudoaxial positions, the slightly stronger shielding in $(\mathbf{3 S}, \mathbf{5 R})-\mathbf{3}$ could be attributed to the anisotropic effect of the phenyl ring present in the spatial vicinity.

The racemic 2,6-DKPs 3f, $\mathbf{g}$ were synthesized from the corresponding $N$-substituted glycines in a similar manner, according to the reaction sequences depicted in Scheme 2. Notably, the chemical yields of racemic U-5C-4CR products 1f and $\mathbf{g}$ (18 and $24 \%$, respectively) were significantly lower than those observed for $N$-unsubstituted adducts $\mathbf{1 a - e}$.

\section{Anticonvulsant screening}

Compounds 3a-f were evaluated in the in vivo animal models of epilepsy within the Anticonvulsant Screening Program (ASP) of The National Institute of Neurological Disorders and Stroke (NINDS), at The National Institutes of Health according to well-established protocols described in the "Experimental" section of this article. The compounds were screened using maximal electroshock seizure (MES) and subcutaneous metrazole (scMET) tests (White et al., 2002). The first of these tests uses an electrical stimulus to induce generalized tonic-clonic seizures and is capable of identifying compounds that prevent the spread of seizure. The latter employs chemically induced seizures to recognize agents that raise the seizure threshold. The most promising compounds were subjected to an evaluation of anticonvulsant activity using a minimal clonic seizure (6 Hz) test (Brown et al., 1953; Barton et al., 2001; Kaminski et al., 2004), which is regarded as a preliminary model of pharmacoresistant limbic seizures. Additionally, a standardized rotorod test for neurological toxicity (TOX; Dunham and Miya, 1957) was performed for each compound. The results are summarized in Tables 1 and 2 .

As shown in Table 1, compounds $3 \mathbf{a}, \mathbf{b}, \mathbf{d}-\mathbf{f}$ exhibited weak to good anticonvulsant activities in the MES model in mice. (3S,5S)-3a and (3S,5S)-3e were most potent, displaying a notable seizure protection in $2 / 3$ and $2 / 4$ of tested 
Fig. 2 Selected nOe correlations in (3S,5S)-3a and $(3 S, 5 R)-3 a$

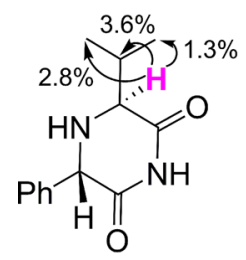

$(3 S, 5 S)-3 a$

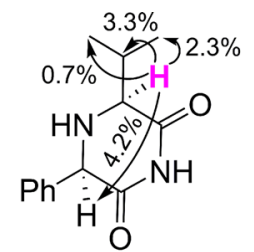<smiles></smiles>

(3S,5R)-3a

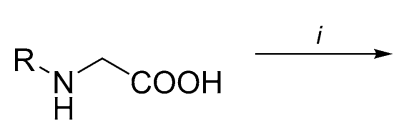

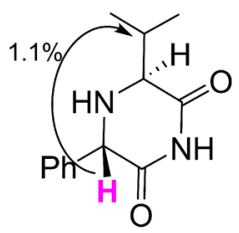

rac-2f,g

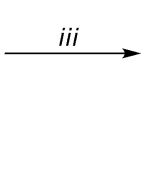

i. $\mathrm{PhCHO}, t \mathrm{BuNC}, \mathrm{MeOH}, \mathrm{FeCl}_{3}$ (cat.), rt, $48 \mathrm{~h}$
ii. $\mathrm{BF}_{3}{ }^{*} 2 \mathrm{CH}_{3} \mathrm{COOH}, 45-55^{\circ} \mathrm{C}, 4-6 \mathrm{~h}$
iii. $\mathrm{NaOH}, \mathrm{EtOH}, \mathrm{tr}, 10 \mathrm{~min}$.

Scheme 2 Synthesis of racemic 2,6-DKP derivatives $\mathbf{3 f}, \mathbf{g}$ animals, respectively, at the dose of $100 \mathrm{mg} / \mathrm{kg}$, after $0.5 \mathrm{~h}$ postadministration. No neurotoxicity was detected at the same dose. $(\mathbf{3 S}, \mathbf{5 S})-\mathbf{3 e}$ also proved effective also at the later time points (1/4 and 4/4 at 100 and $300 \mathrm{mg} / \mathrm{kg}$, respectively, at $2.0 \mathrm{~h}$ ). None of the compounds investigated were significantly active in the scMET model.

According to our previous reports on bicyclic 2,6-DKP derivatives, the $(R, S)$ isomers were completely devoid of any in vivo pharmacological activity (Dawidowski et al., 2011, 2012a). In contrast, the monocyclic derivatives $(\mathbf{3 R}, \mathbf{5} S)-\mathbf{3 a}$ and $(\mathbf{3} R, \mathbf{5} S)-\mathbf{3 e}$, displayed a weak, yet noticeable activity in the MES test $(1 / 1$ and $1 / 4$ at $300 \mathrm{mg}$, respectively, at $0.5 \mathrm{~h}$ ). This could mean that the greater flexibility due to the lack of fused alkyl rings allows for a better fit in the putative binding site within the CNS. Nevertheless, the $(S, S)$ isomers again proved more active.

In general, the most significant levels of seizure protection were observed for derivatives bearing alkyl or aryl substituents at carbon C-5. Among the compounds with alkyl groups, the L-valine derivative with isopropyl side chain $(\mathbf{3 S}, \mathbf{5 S})$-3a was most potent in the MES test. High levels of seizure protection was also observed for symmetrical $(3 S, 5 S)$-3e having two benzene rings with a proper stereochemistry with respect to the 2,6-DKP core. Importantly, the anticonvulsant activity of the investigated molecules was not dependant on their $\log P$ values.

Derivatives (3S,5S)-3a and $(\mathbf{3} S, \mathbf{5} S)-\mathbf{3 e}$ were further assessed for their potential efficacy against pharmacoresistant epilepsy using the $6 \mathrm{~Hz}$ screen. The results are summarized in Table 2 . Notable activity was detected for the first compound $(2 / 4$ and $1 / 4$, at 0.25 and $0.5 \mathrm{~h}$, respectively, at $100 \mathrm{mg} / \mathrm{kg}$ ), while the latter was inactive.

\section{Conclusions}

We have synthesized a series of novel diastereomerically pure, monocyclic 2,6-DKP derivatives by use of diastereoselective synthetic sequence using the U-5C-4CR multicomponent reaction as the key step. The compounds displayed weak to good anticonvulsant activities in the MES model, while none of them were active in scMET screen. The most promising compound (3S,5S)-3a exhibited notable action in the $6 \mathrm{~Hz}$ test. Contrary to the recently reported activity of bicyclic 2,6-DKPs, the activity of monocyclic derivatives seemed to be less stereochemistry-dependent. We conclude that this is due to increased conformational flexibility. Although the seizure-suppressing potency of the newly synthesized agents was generally weaker relative to bicyclic 2,6DKPs, they possess secondary amino groups that provide additional points of diversification for further SAR studies.

\section{Experimental}

\section{Chemistry}

Melting points were determined on an Electrothermal 9100 apparatus in open capillary tubes and were uncorrected. The IR spectra (thin film on $\mathrm{KBr}$ pellets) were recorded on a Shimadzu FTIR-8300 instrument. The NMR spectra were obtained on a Varian Inova $500 \mathrm{MHz}$ spectrometer. Chemical shifts $(\delta)$ were expressed in ppm relative to tetramethylsilane or solvent used as the internal reference. The following abbreviations were used to describe the peak patterns: s (singlet), d (doublet), $\mathrm{t}$ (triplet), q (quartet), qt (quintet), sp (septet), m (multiplet), p (pseudo-), and b 
Table 1 Anticonvulsant activity and neurotoxicity of compounds in the MES and scMET models following intraperitoneal (ip) administration in mice

\begin{tabular}{|c|c|c|c|c|c|c|c|c|}
\hline \multirow[t]{2}{*}{ Compounds } & \multirow{2}{*}{$\begin{array}{l}\text { Dose } \\
(\mathrm{mg} / \mathrm{kg})\end{array}$} & \multicolumn{2}{|c|}{$\mathrm{MES}^{\mathrm{a}}$} & \multicolumn{2}{|c|}{$\mathrm{scMET}^{\mathrm{b}}$} & \multicolumn{2}{|c|}{$\mathrm{TOX}^{\mathrm{c}}$} & \multirow[t]{2}{*}{$C \log P^{\mathrm{c}}$} \\
\hline & & $0.5 \mathrm{~h}$ & $\begin{array}{l}4.0 \\
\mathrm{~h}^{\mathrm{e}}\end{array}$ & $0.5 \mathrm{~h}$ & $4.0 \mathrm{~h}$ & $0.5 \mathrm{~h}$ & $\begin{array}{l}4.0 \\
\mathrm{~h}^{\mathrm{e}}\end{array}$ & \\
\hline \multirow[t]{3}{*}{$(3 S, 5 S)-3 \mathrm{a}$} & 30 & $0 / 1$ & $0 / 1$ & $0 / 1$ & $0 / 1$ & $0 / 4$ & $0 / 2$ & 0.80 \\
\hline & 100 & $2 / 3$ & $0 / 3$ & $0 / 1$ & $0 / 1$ & $0 / 8$ & $0 / 4$ & \\
\hline & 300 & $1 / 1$ & $0 / 1$ & $0 / 1$ & $0 / 1$ & $\mathbf{4}_{\mathrm{g}}^{\mathrm{f}}$ & $0 / 2$ & \\
\hline \multirow[t]{3}{*}{$(3 S, 5 R)-3 a$} & 30 & $0 / 1$ & $0 / 1$ & $0 / 1$ & $0 / 1$ & $0 / 4$ & $0 / 2$ & 0.80 \\
\hline & 100 & $0 / 3^{\mathrm{h}}$ & $0 / 3$ & $1 / 5^{\mathrm{i}}$ & $0 / 1$ & $0 / 8$ & $0 / 4$ & \\
\hline & 300 & $1 / 1$ & $0 / 1$ & $0 / 1$ & $0 / 1$ & $2 / 4$ & $0 / 2$ & \\
\hline \multirow[t]{3}{*}{$(3 S, 5 S)-3 b$} & 30 & $0 / 1$ & $0 / 1$ & $0 / 1$ & $0 / 1$ & $0 / 4$ & $0 / 2$ & 1.19 \\
\hline & 100 & $0 / 3$ & $0 / 3$ & $0 / 1$ & $0 / 1$ & $0 / 8$ & $0 / 4$ & \\
\hline & 300 & $1 / 1$ & $0 / 1$ & $0 / 1$ & $0 / 1$ & $3 / 4^{\mathrm{f}}$ & $0 / 2$ & \\
\hline \multirow[t]{3}{*}{$(3 S, 5 S)-3 \mathrm{c}$} & 30 & $0 / 1$ & $0 / 1$ & $0 / 1$ & $0 / 1$ & $0 / 4$ & $0 / 2$ & 1.19 \\
\hline & 100 & $0 / 3$ & $0 / 3$ & $0 / 1$ & $0 / 1$ & $0 / 8$ & $0 / 4$ & \\
\hline & 300 & $0 / 1$ & $0 / 1$ & $0 / 1$ & $0 / 1$ & $2 / 4$ & $0 / 2$ & \\
\hline \multirow[t]{3}{*}{$(3 S, 5 S)-3 d$} & 30 & $0 / 1$ & $0 / 1$ & $0 / 1$ & $0 / 1$ & $0 / 4$ & $0 / 2$ & 1.61 \\
\hline & 100 & $0 / 3$ & $0 / 3$ & $0 / 1$ & $0 / 1$ & $0 / 8$ & $0 / 4$ & \\
\hline & 300 & $1 / 1$ & $0 / 1$ & $0 / 1$ & $0 / 1$ & $0 / 4$ & $0 / 2$ & \\
\hline \multirow[t]{3}{*}{$(3 S, 5 S)-3 e$} & 30 & $0 / 4$ & $0 / 4$ & - & - & $0 / 8$ & $0 / 8$ & 2.12 \\
\hline & 100 & $2 / 4$ & $1 / 4$ & - & - & $0 / 8$ & $0 / 8$ & \\
\hline & 300 & $4 / 4$ & $4 / 4$ & - & - & $2 / 8$ & $1 / 8$ & \\
\hline \multirow[t]{3}{*}{$(3 S, 5 R)-3 e$} & 30 & $0 / 4$ & $0 / 4$ & - & - & $0 / 8$ & $0 / 8$ & 2.12 \\
\hline & 100 & $0 / 4$ & $0 / 4$ & - & - & $0 / 8$ & $0 / 8$ & \\
\hline & 300 & $1 / 4$ & $0 / 4$ & - & - & $0 / 8$ & $0 / 8$ & \\
\hline \multirow[t]{3}{*}{ rac $-\mathbf{3 f}$} & 30 & $0 / 4$ & $0 / 4$ & - & - & $0 / 8$ & $0 / 8$ & 2.29 \\
\hline & 100 & $0 / 4$ & $0 / 4$ & - & - & $0 / 8$ & $0 / 8$ & \\
\hline & 300 & $0 / 4$ & $3 / 4$ & - & - & $0 / 8$ & $0 / 8$ & \\
\hline \multirow[t]{3}{*}{$r a c-3 g$} & 30 & $0 / 1$ & $0 / 1$ & $0 / 1$ & $0 / 1$ & $0 / 4$ & $0 / 2$ & 2.12 \\
\hline & 100 & $0 / 3$ & $0 / 3$ & $0 / 1$ & $0 / 1$ & $0 / 8$ & $0 / 4$ & \\
\hline & 300 & $0 / 1$ & $0 / 1$ & $0 / 1$ & $0 / 1$ & $0 / 4$ & $0 / 2$ & \\
\hline
\end{tabular}

Ratios where at least one animal was protected or displayed neurotoxicity have been highlighted in bold to enhance data readability and interpretation

${ }^{a}$ Maximal electroshock test (number of animals protected/number of animals tested)

${ }^{b}$ Subcutaneous metrazole test (number of animals protected/number of animals tested)

${ }^{c}$ Neurotoxicity test (number of animals exhibiting neurological toxicity/number of animals tested)

d Theoretical $\log P$ value calculated by a $\log$ arithm included in $\mathrm{Hy}-$ perChem 7.5 package

e Compounds (3S,5S)-3e, $(\mathbf{3 S}, \mathbf{5 R})-\mathbf{3 e}$ and rac-3f were tested at $2.0 \mathrm{~h}$ post administration

${ }^{\mathrm{f}}$ Unable to grasp rotorod

g Loss of righting reflex

$\mathrm{h}$ Active also in $1 / 3$ at $0.25 \mathrm{~h}$ post administration

${ }^{\mathrm{i}}$ Myoclonic jerks
Table 2 Anticonvulsant activity and neurotoxicity of compounds in the $6 \mathrm{~Hz}$ model following intraperitoneal (ip) administration in mice

\begin{tabular}{lllllll}
\hline Compounds & Test $^{\mathrm{a}}$ & $0.25 \mathrm{~h}$ & $0.5 \mathrm{~h}$ & $1.0 \mathrm{~h}$ & $2.0 \mathrm{~h}$ & $4.0 \mathrm{~h}$ \\
\hline $\mathbf{( 3 S , 5 S ) - 3 a}$ & $6 \mathrm{~Hz}^{\mathrm{b}}$ & $\mathbf{2 / 4}$ & $\mathbf{1 / 4}$ & $0 / 4$ & $0 / 4$ & $0 / 4$ \\
& $\mathrm{TOX}^{\mathrm{c}}$ & $0 / 4$ & $0 / 4$ & $0 / 4$ & $0 / 4$ & $0 / 4$ \\
$\mathbf{( 3 S , 5 S ) - 3 e}$ & $6 \mathrm{~Hz}$ & - & $0 / 4$ & - & $0 / 4$ & - \\
& $\mathrm{TOX}$ & - & $0 / 8$ & - & $0 / 8$ & - \\
\hline
\end{tabular}

Ratios where at least one animal was protected or displayed neurotoxicity have been highlighted in bold to enhance data readability and interpretation

a At dose $100 \mathrm{mg} / \mathrm{kg}$

${ }^{\text {b }} 6 \mathrm{~Hz}$ test, $32 \mathrm{~mA}$ (number of animals protected/number of animals tested)

c Neurotoxicity test (number of animals exhibiting neurological toxicity/number of animals tested)

(broad-). Coupling constants $(J)$ were in hertz $(\mathrm{Hz})$. The electrospray ionization high-resolution mass spectra (ESIHRMS) were recorded on a LCT TOF (Micromass) instrument. Optical rotations were measured with a Perkin-Elmer 241 polarimeter at $20^{\circ} \mathrm{C}$, using a sodium lamp (589 nm). Thin-layer chromatography (TLC) was run on Merck Silica gel-60 $\mathrm{F}_{254}$ plates. The spots were visualized by ultraviolet light $(254 \mathrm{~nm})$ or iodine vapors. Flash column chromatography (FC) was carried out on Merck Silica gel 60 (particle size $0.040-0.063 \mathrm{~mm}$ ). Solvents were dried and purified by standard methods. Petroleum ether (PE) referred to the fraction boiling at $40-60{ }^{\circ} \mathrm{C}$. All reagents were purchased from commercial sources and used as received. Unless otherwise stated, the chemical yields were calculated for pure $\left(d_{\mathrm{r}} \geq 95 / 5\right)$ compounds. Compound rac-3g was synthesized as described previously (Dawidowski et al., 2012b).

Synthesis of compounds 1 by U-5C-4CR condensation

Iron(III) chloride (5 mol.\%) and tert-butyl isocyanide (1.0 equiv.) were added to a stirred suspension of appropriate $\alpha$-amino acid (1.2 equiv.) and benzaldehyde (1.0 equiv.) in $\mathrm{MeOH}(100 \mathrm{~mL})$. The mixture was stirred at RT for $48 \mathrm{~h}$ and the volatiles were removed under reduced pressure. The resulting crude products were purified FC.

Methyl (2S, 1S)- and (2S,1R)-2-(2-(tert-butylamino)-2oxo-1-phenylethylamino)-3-methylbutanoate $(2 S, 1 S)-1$ a and $(2 S, 1 R)-1 a$

From L-valine $(2.36 \mathrm{~g}, 20.16 \mathrm{mmol})$, benzaldehyde (16.80 mmol, $1.71 \mathrm{~mL})$ and tert-butyl isocyanide $(2.00 \mathrm{~mL}$, $16.80 \mathrm{mmol}$ ); FC (gradient: PE/AcOEt 6:1-3:1): yield $4.04 \mathrm{~g}(75 \%)$ of chromatographically inseparable 
diastereomeric mixture $\left(d_{\mathrm{r}}=7.3 / 1,{ }^{1} \mathrm{H}\right.$ NMR). Analytical sample of $(2 S, 1 S)$-1a was obtained by recrystallization from $\mathrm{PE} / \mathrm{Et}_{2} \mathrm{O}$ 10:1. (2S,1S)-1a: white wax; $\mathrm{mp} 37-38{ }^{\circ} \mathrm{C}$; $[\alpha]_{\mathrm{D}}=-97.2\left(c 1, \mathrm{CHCl}_{3}\right)$; IR (KBr): 729, 764, 1200, 1454, 1516, 1678, 1736, 2874, 2962, 3333; TLC (PE/AcOEt 3:1): $R_{\mathrm{f}}=0.43 ;{ }^{1} \mathrm{H} \mathrm{NMR}\left(\mathrm{CDCl}_{3}, 500 \mathrm{MHz}\right): \delta 0.89\left(\mathrm{~d},{ }^{3} J=6.5\right.$, $\left.3 \mathrm{H}, \mathrm{CH}_{3}\right), 0.93\left(\mathrm{~d},{ }^{3} \mathrm{~J}=6.5,3 \mathrm{H}, \mathrm{CH}_{3}^{\prime}\right), 1.29(\mathrm{~s}, 9 \mathrm{H}$, $\left.\mathrm{C}\left(\mathrm{CH}_{3}\right)_{3}\right), 1.96\left(\mathrm{~m},{ }^{3} \mathrm{~J}=6.5,1 \mathrm{H}, \mathrm{CH}\right), 2.34(\mathrm{bs}, 1 \mathrm{H}, \mathrm{NH})$, $2.87\left(\mathrm{bpd},{ }^{3} \mathrm{~J}=5.0,1 \mathrm{H}, \mathrm{H}-2\right), 3.71\left(\mathrm{~s}, 3 \mathrm{H}, \mathrm{OCH}_{3}\right), 4.08(\mathrm{~s}$, $1 \mathrm{H}, \mathrm{H}-1), 6.37$ (bs, $1 \mathrm{H}, \mathrm{CONH}$ ), 7.28-7.36 (m, 5H, H-Ar); ${ }^{13} \mathrm{C} \mathrm{NMR}\left(\mathrm{CDCl}_{3}, 125 \mathrm{MHz}\right): \delta 18.4\left(\mathrm{CH}_{3}\right), 19.2\left(\mathrm{CH}_{3}^{\prime}\right)$,

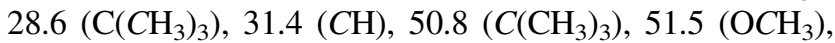
$64.6(\mathrm{C}-2), 66.6(\mathrm{C}-1), 127.9\left(\mathrm{C}-2^{\prime}, \mathrm{C}-6^{\prime}\right), 128.2\left(\mathrm{C}-4^{\prime}\right), 128.8$ $\left(\mathrm{C}-3^{\prime}, \mathrm{C}-5^{\prime}\right), 138.8\left(\mathrm{C}-1^{\prime}\right), 170.9(\mathrm{CONH}), 174.7\left(\mathrm{COOCH}_{3}\right)$; HRMS (ESI) calcd for $\mathrm{C}_{18} \mathrm{H}_{28} \mathrm{~N}_{2} \mathrm{O}_{3} \mathrm{Na}$ : $343.1998(\mathrm{M}+\mathrm{Na})^{+}$ found 343.1958. (2S,1R)-1a: ${ }^{1} \mathrm{H}$ NMR (from diastereomeric mixture, $\left.\mathrm{CDCl}_{3}, 500 \mathrm{MHz}\right): 0.95\left(\mathrm{~d},{ }^{3} \mathrm{~J}=6.5,3 \mathrm{H}, \mathrm{CH}_{3}\right)$, $1.06\left(\mathrm{~d},{ }^{3} J=6.5,3 \mathrm{H}, \mathrm{CH} H_{3}^{\prime}\right), 1.39\left(\mathrm{~s}, 9 \mathrm{H}, \mathrm{C}\left(\mathrm{CH}_{3}\right)_{3}\right), 2.02(\mathrm{~m}$, $\left.{ }^{3} J=6.5,1 \mathrm{H}, \mathrm{CH}\right), 2.34$ (bs, $\left.1 \mathrm{H}, \mathrm{NH}\right), 3.09$ (m, 1H, H-2), 3.73 (s, $3 \mathrm{H}, \mathrm{OCH}_{3}$ ), 3.92 (s, $1 \mathrm{H}, \mathrm{H}-1$ ), 6.37 (bs, $1 \mathrm{H}, \mathrm{CONH}$ ), the remaining signals overlap with the signals of $(2 S, 1 S)-1 a$; ${ }^{13} \mathrm{C} \mathrm{NMR}$ (from diastereomeric mixture, $\mathrm{CDCl}_{3}, 125 \mathrm{MHz}$ ): $\delta 18.0\left(\mathrm{CH}_{3}\right), 19.6\left(\mathrm{CH}_{3}^{\prime}\right), 28.8\left(\mathrm{C}\left(\mathrm{CH}_{3}\right)_{3}\right), 31.5(\mathrm{CH}), 50.7$ $\left(\mathrm{C}\left(\mathrm{CH}_{3}\right)_{3}\right), 51.8\left(\mathrm{OCH}_{3}\right), 66.3(\mathrm{C}-1), 67.0(\mathrm{C}-2), 127.3\left(\mathrm{C}-2^{\prime}\right.$, C-6'), $128.3\left(\mathrm{C}-4^{\prime}\right), 128.8\left(\mathrm{C}-3^{\prime}, \mathrm{C}-5^{\prime}\right), 139.6\left(\mathrm{C}-1^{\prime}\right), 170.9$ $(\mathrm{CONH}), 175.0\left(\mathrm{COOCH}_{3}\right)$.

\section{Methyl (2S, 1S)- and (2S,1R)-2-(2-(tert-butylamino)-2- oxo-1-phenylethylamino)-4-methylpentanoate (2S,1S)-1b and $(2 S, 1 R)-1 b$}

From L-leucine $(2.64 \mathrm{~g}, 20.16 \mathrm{mmol})$, benzaldehyde $(16.80 \mathrm{mmol}, 1.71 \mathrm{~mL})$ and tert-butyl isocyanide $(2.00 \mathrm{~mL}$, $16.80 \mathrm{mmol}$ ); FC (gradient: PE/AcOEt 9:1-4:1): yield $3.58 \mathrm{~g}(64 \%)$ of diastereomeric mixture $\left(d_{\mathrm{r}}=5.3 / 1,{ }^{1} \mathrm{H}\right.$ NMR). Colorless oil; IR (KBr): 700, 733, 1155, 1200, 1227 , 1454, 1516, 1680, 1738, 2870, 2959, 3331; TLC (PE/AcOEt 3:1): $R_{\mathrm{f}}=0.35$ (major isomer) and 0.38 (minor isomer); ${ }^{1} \mathrm{H}$ NMR (from diastereomeric mixture, $\mathrm{CDCl}_{3}, 500 \mathrm{MHz}$ ): (2S,1S)-1b (major isomer): $\delta 0.77\left(\mathrm{~d},{ }^{3} \mathrm{~J}=6.5,3 \mathrm{H}, \mathrm{CH}_{3}\right)$, $0.87\left(\mathrm{~d},{ }^{3} \mathrm{~J}=6.5,3 \mathrm{H}, \mathrm{CH}_{3}^{\prime}\right), 1.31\left(\mathrm{~s}, 9 \mathrm{H}, \mathrm{C}\left(\mathrm{CH}_{3}\right)_{3}\right), 1.58(\mathrm{~m}$, $\left.2 \mathrm{H}, \mathrm{CH}_{2}\right), 1.71\left(\mathrm{~m},{ }^{3} J=6.5,1 \mathrm{H}, \mathrm{CH}\right), 2.26(\mathrm{bs}, 1 \mathrm{H}, \mathrm{NH})$, $3.11\left(\mathrm{pt}^{3}{ }^{3} \mathrm{~J}=7.5,1 \mathrm{H}, \mathrm{H}-2\right), 3.70\left(\mathrm{~s}, 3 \mathrm{H}, \mathrm{OCH}_{3}\right), 4.11(\mathrm{~s}, 1 \mathrm{H}$, $\mathrm{H}-1$ ), 6.49 (bs, 1H, CONH), 7.28-7.37 (m, 5H, H-Ar); (2S,1R)-1b (minor isomer): $\delta 0.96\left(\mathrm{~d},{ }^{3} \mathrm{~J}=6.5,3 \mathrm{H}, \mathrm{CH}_{3}\right)$, $0.99\left(\mathrm{~d},{ }^{3} \mathrm{~J}=6.5,3 \mathrm{H}, \mathrm{CH}_{3}^{\prime}\right), 1.38\left(\mathrm{~s}, 9 \mathrm{H}, \mathrm{C}\left(\mathrm{CH}_{3}\right)_{3}\right), 1.86(\mathrm{~m}$, $\left.{ }^{3} J=6.5,1 \mathrm{H}, \mathrm{CH}\right), 3.32\left(\mathrm{dd},{ }^{3} J_{1}=9.0,{ }^{3} J_{2}=5.0,1 \mathrm{H}, \mathrm{H}-2\right)$, $3.72\left(\mathrm{~s}, 3 \mathrm{H}, \mathrm{OCH}_{3}\right), 3.95(\mathrm{~s}, 1 \mathrm{H}, \mathrm{H}-1)$, the remaining signals overlap with the signals of $(2 S, 1 S)-1 \mathbf{b} ;{ }^{13} \mathrm{C}$ NMR (from diastereomeric mixture, $\left.\mathrm{CDCl}_{3}, 125 \mathrm{MHz}\right):(\mathbf{2 S}, \mathbf{1 S})-\mathbf{1 b}$ (major isomer): $\delta 22.0\left(\mathrm{CH}_{3}\right), 22.8\left(\mathrm{CH}_{3}^{\prime}\right), 24.8(\mathrm{CH}), 28.6$

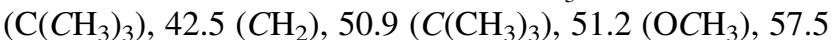
(C-2), 66.4 (C-1), $127.8\left(\mathrm{C}-2^{\prime}, \mathrm{C}^{\prime} 6^{\prime}\right), 128.2\left(\mathrm{C}-4^{\prime}\right), 128.9$
$\left(\mathrm{C}-3^{\prime}, \mathrm{C}-5^{\prime}\right), 139.0\left(\mathrm{C}-1^{\prime}\right), 170.8(\mathrm{CONH}), 175.4\left(\mathrm{COOCH}_{3}\right)$; (2S,1R)-1b (minor isomer): $\delta 22.2\left(\mathrm{CH}_{3}\right), 23.2\left(\mathrm{CH}_{3}^{\prime}\right), 24.9$ $(\mathrm{CH}), 28.7\left(\mathrm{C}\left(\mathrm{CH}_{3}\right)_{3}\right), 43.4\left(\mathrm{CH}_{2}\right), 50.7\left(\mathrm{C}_{\left.\left(\mathrm{CH}_{3}\right)_{3}\right), 52.0}\right.$ $\left(\mathrm{OCH}_{3}\right), 59.0(\mathrm{C}-2), 66.9(\mathrm{C}-1), 127.2\left(\mathrm{C}-2^{\prime}, \mathrm{C}-6^{\prime}\right), 128.1(\mathrm{C}-$ $\left.4^{\prime}\right), 128.8\left(\mathrm{C}-3^{\prime}, \mathrm{C}-5^{\prime}\right), 139.9\left(\mathrm{C}-1^{\prime}\right), 170.9(\mathrm{CONH}), 175.9$ $\left(\mathrm{COOCH}_{3}\right)$; HRMS (ESI) calcd for $\mathrm{C}_{18} \mathrm{H}_{28} \mathrm{~N}_{2} \mathrm{O}_{3} \mathrm{Na}$ : $357.2154(\mathrm{M}+\mathrm{Na})^{+}$found 357.2171.

Methyl (2S, $1 S, 3 S)$ - and (2S,1R,3S)-2-(2-(tert-butylamino)2-oxo-1-phenylethylamino)-3-methylpentanoate $(2 S, 1 S, 3 S)-1 c$ and $(2 S, 1 R, 3 S)-1 c$

From L-isoleucine $(2.64 \mathrm{~g}, 20.16 \mathrm{mmol})$, benzaldehyde $(16.80 \mathrm{mmol}, \quad 1.71 \mathrm{~mL})$ and tert-butyl isocyanide (2.00 mL, $16.80 \mathrm{mmol})$; FC (gradient: PE/AcOEt 9:1-4:1): yield $3.97 \mathrm{~g}(71 \%)$ of chromatographically inseparable diastereomeric mixture $\left(d_{\mathrm{r}}=9.0 / 1,{ }^{1} \mathrm{H}\right.$ NMR $)$. Colorless oil; IR (KBr): 700, 741, 1148, 1200, 1225, 1265, 1454, 1516, 1678, 1736, 2876, 2930, 2964, 3329; TLC (PE/ AcOEt 3:1): $R_{\mathrm{f}}=0.35 ;{ }^{1} \mathrm{H}$ NMR (from diastereomeric mixture, $\left.\mathrm{CDCl}_{3}, 500 \mathrm{MHz}\right)$ : $(\mathbf{2 S}, \mathbf{1 S}, 3 \mathrm{~S})-\mathbf{1 c}$ (major isomer): $\delta 0.83\left(\mathrm{t},{ }^{3} \mathrm{~J}=7.5,3 \mathrm{H}, \mathrm{CH}_{2} \mathrm{CH}_{3}\right), 0.85\left(\mathrm{~d},{ }^{3} \mathrm{~J}=7.0,3 \mathrm{H}\right.$, $\left.\mathrm{CH}_{3}\right), 1.16\left(\mathrm{~m}, 1 \mathrm{H}, \mathrm{CH}_{2}\right), 1.30\left(\mathrm{~s}, 9 \mathrm{H}, \mathrm{C}\left(\mathrm{CH}_{3}\right)_{3}\right), 1.51(\mathrm{~m}$, $\left.1 \mathrm{H}, \mathrm{CH}_{2}^{\prime}\right), 1.72(\mathrm{~m}, 1 \mathrm{H}, \mathrm{CH}), 2.35(\mathrm{bs}, 1 \mathrm{H}, \mathrm{NH}), 2.94(\mathrm{~d}$, $\left.{ }^{3} J=6.0,1 \mathrm{H}, \mathrm{H}-2\right), 3.71\left(\mathrm{~s}, 3 \mathrm{H}, \mathrm{OCH}_{3}\right), 4.07(\mathrm{~s}, 1 \mathrm{H}, \mathrm{H}-1)$, $6.37(\mathrm{bs}, \quad 1 \mathrm{H}, \mathrm{CONH}), \quad 7.27-7.34(\mathrm{~m}, 5 \mathrm{H}, \mathrm{H}-\mathrm{Ar})$; $(\mathbf{2 S}, \mathbf{1 R}, 3 \mathrm{~S})-1 \mathrm{c}$ (minor isomer): $\delta 0.92\left(\mathrm{t},{ }^{3} \mathrm{~J}=7.5,3 \mathrm{H}\right.$, $\left.\mathrm{CH}_{2} \mathrm{CH}_{3}\right), 1.00$ (d, $\left.{ }^{3} \mathrm{~J}=7.0,3 \mathrm{H}, \mathrm{CH}_{3}\right), 1.39(\mathrm{~s}, 9 \mathrm{H}$, $\left.\mathrm{C}\left(\mathrm{CH}_{3}\right)_{3}\right), 3.17\left(\mathrm{~d},{ }^{3} \mathrm{~J}=5.5,1 \mathrm{H}, \mathrm{H}-2\right), 3.72\left(\mathrm{~s}, 3 \mathrm{H}, \mathrm{OCH}_{3}\right)$, $3.93(\mathrm{~s}, 1 \mathrm{H}, \mathrm{H}-1), 5.30(\mathrm{bs}, 1 \mathrm{H}, \mathrm{CON} H)$, the remaining signals overlap with the signals of $(2 S, 1 S, 3 S)-1 \mathrm{c} ;{ }^{13} \mathrm{C} N M R$ (from diastereomeric mixture, $\mathrm{CDCl}_{3}, 125 \mathrm{MHz}$ ): (2S,1S,3S)-1c (major isomer): $\delta$ 11.3, $15.6\left(\mathrm{CH}_{3}, \mathrm{CH}_{3}^{\prime}\right)$,

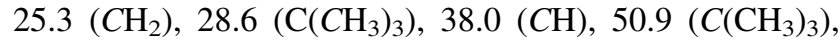
$51.5\left(\mathrm{OCH}_{3}\right), 63.5(\mathrm{C}-2), 66.6(\mathrm{C}-1), 127.9\left(\mathrm{C}-2^{\prime}, \mathrm{C}^{\prime} 6^{\prime}\right)$, $128.2\left(\mathrm{C}-4^{\prime}\right), 128.8\left(\mathrm{C}-3^{\prime}, \mathrm{C}^{\prime} 5^{\prime}\right), 138.8\left(\mathrm{C}-1^{\prime}\right), 170.9$ (CONH), $174.7\left(\mathrm{COOCH}_{3}\right) ;(\mathbf{2 S , 1 R , 3 S ) - 1 c}$ (minor isomer): $\delta$ 11.7, $16.4\left(\mathrm{CH}_{3}, \mathrm{CH}_{3}^{\prime}\right), 25.0\left(\mathrm{CH}_{2}\right), 28.8\left(\mathrm{C}_{\left.\left(\mathrm{CH}_{3}\right)_{3}\right), 38.5}\right.$

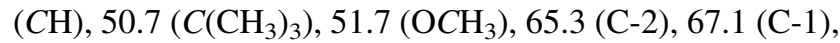
$127.2\left(\mathrm{C}-2^{\prime}, \mathrm{C}^{\prime} 6^{\prime}\right), 128.0\left(\mathrm{C}-4^{\prime}\right), 128.8\left(\mathrm{C}-3^{\prime}, \mathrm{C}^{\prime} 5^{\prime}\right), 139.6$ $\left(\mathrm{C}-1^{\prime}\right), 171.0(\mathrm{CONH}), 174.7\left(\mathrm{COOCH}_{3}\right)$; HRMS (ESI) calcd for $\mathrm{C}_{18} \mathrm{H}_{28} \mathrm{~N}_{2} \mathrm{O}_{3} \mathrm{Na}$ : $357.2154(\mathrm{M}+\mathrm{Na})^{+}$found 357.2148 .

Methyl (2S, 1S)- and (2S,1R)-2-(2-(tert-butylamino)-2oxo-1-phenylethylamino)-3-phenylpropanoate (2S,1S)-1d and $(2 S, 1 R)-1 d$

From L-phenylalanine ( $3.33 \mathrm{~g}, 20.16 \mathrm{mmol}$ ), benzaldehyde $(16.80 \mathrm{mmol}, \quad 1.71 \mathrm{~mL})$ and tert-butyl isocyanide (2.00 mL, $16.80 \mathrm{mmol}$ ); FC (gradient: PE/AcOEt 9:1-2:1): yield $3.23 \mathrm{~g}(52 \%)$ of diastereomeric mixture $\left(d_{\mathrm{r}}=5.1 / 1\right.$, ${ }^{1}$ H NMR). Pale-yellow oil; IR (KBr): 700, 754, 1223, 1454, 
1516, 1680, 1738, 2872, 2966, 3326; TLC (PE/AcOEt 3:1): $R_{\mathrm{f}}=0.20$ (major isomer) and 0.24 (minor isomer); ${ }^{1} \mathrm{H}$ $\mathrm{NMR}$ (from diastereomeric mixture, $\mathrm{CDCl}_{3}, 500 \mathrm{MHz}$ ): (2S,1S)-1d (major isomer): $\delta 1.28\left(\mathrm{~s}, 9 \mathrm{H}, \mathrm{C}\left(\mathrm{CH}_{3}\right)_{3}\right), 2.33$ (bs, $1 \mathrm{H}, \mathrm{NH}), 2.85\left(\mathrm{dd},{ }^{2} J=13.5,{ }^{3} J=8.0,1 \mathrm{H}, \mathrm{CH}_{2}\right.$ ), $3.03\left(\mathrm{dd},{ }^{2} J=13.5,{ }^{3} J=6.0,1 \mathrm{H}, \mathrm{CH}_{2}^{\prime}\right), 3.36(\mathrm{dd}$, $\left.{ }^{3} J=8.0,{ }^{3} J=6.0,1 \mathrm{H}, \mathrm{H}-2\right), 3.68\left(\mathrm{~s}, 3 \mathrm{H}, \mathrm{OCH}_{3}\right), 4.08(\mathrm{~s}$, 1H, H-1), 6.67 (bs, 1H, CONH), 7.06 (m, 2H, H-Ar), 7.10 (m, 2H, H-Ar), 7.21-7.37 (m, 6H, H-Ar); (2S,1R)-1d (minor isomer): $\delta 1.08\left(\mathrm{~s}, 9 \mathrm{H}, \mathrm{C}\left(\mathrm{CH}_{3}\right)_{3}\right), 2.68(\mathrm{dd}$, $\left.{ }^{2} J=13.5,{ }^{3} J=10.0,1 \mathrm{H}, \mathrm{CH}_{2}\right), 3.47 \quad\left(\mathrm{dd},{ }^{3} J=10.0\right.$, $\left.{ }^{3} J=4.0,1 \mathrm{H}, \mathrm{H}-2\right), 3.75$ (s, 3H, OCH $H_{3}, 3.96$ (s, 1H, H-1), 6.78 (bs, $1 \mathrm{H}, \mathrm{CONH}$ ), the remaining signals overlap with the signals of $\left(\mathbf{2 S , 1 S ) - 1 d ; ~}{ }^{13} \mathrm{C} \mathrm{NMR}\right.$ (from diastereomeric mixture, $\left.\mathrm{CDCl}_{3}, 125 \mathrm{MHz}\right)$ : $(\mathbf{2 S , 1 S ) - 1 d}$ (major isomer): $\delta$ $28.6\left(\mathrm{C}\left(\mathrm{CH}_{3}\right)_{3}\right), 39.4\left(\mathrm{CH}_{2}\right), 50.8\left(\mathrm{C}\left(\mathrm{CH}_{3}\right)_{3}\right), 51.9\left(\mathrm{OCH}_{3}\right)$, 60.4 (C-2), 66.4 (C-1), $126.8\left(\mathrm{C}-4^{\prime \prime}\right), 127.6\left(\mathrm{C}-2^{\prime}, \mathrm{C}-6^{\prime}\right)$, $128.1\left(\mathrm{C}-4^{\prime}\right), 128.5\left(\mathrm{C}-2^{\prime \prime}, \mathrm{C}-6^{\prime \prime}\right), 128.7\left(\mathrm{C}-3^{\prime}, \mathrm{C}-5^{\prime}\right), 129.3$ $\left(\mathrm{C}-3^{\prime \prime}, \mathrm{C}-5^{\prime \prime}\right), 137.0\left(\mathrm{C}-1^{\prime \prime}\right), 138.4\left(\mathrm{C}-1^{\prime}\right), 170.7$ (CONH), $174.1\left(\mathrm{COOCH}_{3}\right)$; (2S,1R)-1d (minor isomer): $\delta 28.4$ $\left(\mathrm{C}\left(\mathrm{CH}_{3}\right)_{3}\right), 40.2\left(\mathrm{CH}_{2}\right), 50.3\left(\mathrm{C}\left(\mathrm{CH}_{3}\right)_{3}\right), 52.1\left(\mathrm{OCH}_{3}\right), 62.4$ (C-2), 66.8 (C-1), 127.0 (C-4"), 127.2 (C-2', C-6'), 128.1 $\left(\mathrm{C}-4^{\prime}\right), 128.7\left(\mathrm{C}-2^{\prime \prime}, \mathrm{C}-6^{\prime \prime}\right), 128.8\left(\mathrm{C}-3^{\prime}, \mathrm{C}-5^{\prime}\right), 129.5\left(\mathrm{C}-3^{\prime \prime}\right.$, $\left.\mathrm{C}-5^{\prime \prime}\right), 137.6\left(\mathrm{C}-1^{\prime \prime}\right), 139.5\left(\mathrm{C}-1^{\prime}\right), 170.5(C \mathrm{ONH}), 174.8$ $\left(\mathrm{COOCH}_{3}\right)$; HRMS (ESI+) calcd for $\mathrm{C}_{22} \mathrm{H}_{28} \mathrm{~N}_{2} \mathrm{O}_{3} \mathrm{Na}$ : $391.1998(\mathrm{M}+\mathrm{Na})^{+}$found 391.1995.

\section{Methyl (2S,1S)- and (2S,1R)-2-(2-(tert-butylamino)-2- oxo-1-phenylethylamino)-3-phenylacetate $(2 S, 1 S)-1 e$ and $(2 S, 1 R)-1 e$}

From L-phenylglycine (3.05 g, $20.16 \mathrm{mmol})$, benzaldehyde (16.80 mmol, $1.71 \mathrm{~mL})$ and tert-butyl isocyanide (2.00 mL, $16.80 \mathrm{mmol}$ ); FC (gradient: PE/AcOEt 5:1-1:2): yield $2.41 \mathrm{~g}(41 \%)$ of chromatographically inseparable diastereomeric mixture $\left(d_{\mathrm{r}}=1.5 / 1,{ }^{1} \mathrm{H}\right.$ NMR). Pale-yellow wax; mp 65-71 ${ }^{\circ} \mathrm{C}$; IR (KBr): 700, 733, 1223, 1454, 1516, 1678, 1740, 2872, 2930, 2966, 3333; TLC (PE/AcOEt 3:1): $R_{\mathrm{f}}=0.28 ;{ }^{1} \mathrm{H} \mathrm{NMR}$ (from diastereomeric mixture, $\mathrm{CDCl}_{3}$, $500 \mathrm{MHz}$ ): (2S,1S)-1e (major isomer): $\delta 1.35$ (s, 9H, $\left.\mathrm{C}\left(\mathrm{CH}_{3}\right)_{3}\right), 2.85$ (bs, $\left.1 \mathrm{H}, \mathrm{NH}\right), 3.69$ (s, 3H, OCH$), 3.99$ (s, $1 \mathrm{H}, \mathrm{H}-1), 4.33$ (s, 1H, H-2), 6.88 (bs, 1H, CONH), 7.23-7.38 (m, 10H, H-Ar); (2S,1R)-1e (minor isomer): $\delta$ 1.27 (s, 9H, $\left.\mathrm{C}\left(\mathrm{CH}_{3}\right)_{3}\right), 2.78$ (bs, 1H, $\left.\mathrm{NH}\right), 3.69$ (s, 3H, $\mathrm{OCH}_{3}$ ), 4.05 (s, 1H, H-1), 4.29 (s, 1H, H-2), 6.97 (bs, 1H, $\mathrm{CONH}$ ); the remaining signals overlap with the signals of (2S,1S)-1e; ${ }^{13} \mathrm{C}$ NMR (from diastereomeric mixture, $\left.\mathrm{CDCl}_{3}, 125 \mathrm{MHz}\right)$ : (2S,1S)-1e (major isomer): $\delta 28.7$ $\left(\mathrm{C}\left(\mathrm{CH}_{3}\right)_{3}\right), 50.9\left(\mathrm{C}\left(\mathrm{CH}_{3}\right)_{3}\right), 52.5\left(\mathrm{OCH}_{3}\right), 63.6(\mathrm{C}-2), 65.1$ (C-1), 127.5, 127.6 (C-2', C-6', C-2", C-6" $\left.{ }^{\prime \prime}\right), 128.2,128.5$ $\left(\mathrm{C}-4^{\prime}, \mathrm{C}-4^{\prime \prime}\right), 128.9,129.0\left(\mathrm{C}-3^{\prime}, \mathrm{C}-5^{\prime}, \mathrm{C}-3^{\prime \prime}, \mathrm{C}-5^{\prime \prime}\right), 137.2$, $139.1\left(\mathrm{C}-1^{\prime}, \mathrm{C}-1^{\prime \prime}\right), 170.5(\mathrm{CONH}), 172.6\left(\mathrm{COOCH}_{3}\right)$; (2S,1R)-1e (minor isomer): $\delta 28.6 \quad\left(\mathrm{C}\left(\mathrm{CH}_{3}\right)_{3}\right), \quad 50.7$
$\left(\mathrm{C}\left(\mathrm{CH}_{3}\right)_{3}\right), 52.4\left(\mathrm{OCH}_{3}\right), 64.1(\mathrm{C}-2), 66.9(\mathrm{C}-1), 127.3$, $127.5\left(\mathrm{C}-2^{\prime}, \mathrm{C}-6^{\prime}, \mathrm{C}-2^{\prime \prime}, \mathrm{C}-6^{\prime \prime}\right), 128.2,128.4\left(\mathrm{C}-4^{\prime}, \mathrm{C}-4^{\prime \prime}\right)$,

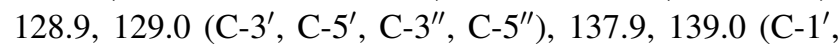
$\left.\mathrm{C}-1^{\prime \prime}\right), 170.6(\mathrm{CONH}), 173.2\left(\mathrm{COOCH}_{3}\right)$; HRMS (ESI+) calcd for $\mathrm{C}_{21} \mathrm{H}_{26} \mathrm{~N}_{2} \mathrm{O}_{3} \mathrm{Na}: 377.1841(\mathrm{M}+\mathrm{Na})^{+}$found 377.1843 .

Methyl (+/-)-2-(2-benzyl-2-(tert-butylamino)-2-oxo-1phenylethylamino)-acetate rac-1f

From $N$-benzylglycine hydrochloride (4.06 g, $20.16 \mathrm{mmol}$ ), triethylamine $(2.81 \mathrm{~mL}, \quad 20.16 \mathrm{mmol})$ benzaldehyde (16.80 mmol, $1.71 \mathrm{~mL})$ and tert-butyl isocyanide $(2.00 \mathrm{~mL}$, $16.80 \mathrm{mmol}$ ); FC (gradient: PE/AcOEt 10:1-3:1): yield $0.77 \mathrm{~g}(12 \%)$. White powder; mp 87-89 ${ }^{\circ} \mathrm{C}$; TLC (PE/ AcOEt 3:1): $R_{\mathrm{f}}=0.40$; IR (KBr): 700, 741, 1204, 1454, $1512,1680,1742,2872,2928,2964,3327 ;{ }^{1} \mathrm{H} \mathrm{NMR}\left(\mathrm{CDCl}_{3}\right.$, $500 \mathrm{MHz}): \delta 1.38\left(\mathrm{~s}, 9 \mathrm{H}, \mathrm{C}\left(\mathrm{CH}_{3}\right)_{3}\right), 3.06\left(\mathrm{~d},{ }^{2} J=17.5,1 \mathrm{H}\right.$, $\left.\mathrm{PhCH}_{2}\right), 3.31\left(\mathrm{~d},{ }^{2} \mathrm{~J}=17.5,1 \mathrm{H}, \mathrm{PhCH}_{2}^{\prime}\right), 3.59(\mathrm{~s}, 3 \mathrm{H}$, $\left.\mathrm{OCH}_{3}\right), 3.67\left(\mathrm{~d},{ }^{2} \mathrm{~J}=13.5,1 \mathrm{H}, \mathrm{CH}_{2}\right), 3.85\left(\mathrm{~d},{ }^{2} \mathrm{~J}=13.5\right.$, $1 \mathrm{H}, \mathrm{CH}_{2}^{\prime}$ ), 4.43 (s, 1H, H-1), 7.26-7.39 (m, 10H, H-Ar), 7.60 (bs, $1 \mathrm{H}, \mathrm{CONH}) ;{ }^{13} \mathrm{C} \mathrm{NMR}\left(\mathrm{CDCl}_{3}, 125 \mathrm{MHz}\right): \delta 28.7$ $\left(\mathrm{C}\left(\mathrm{CH}_{3}\right)_{3}\right), 50.9\left(\mathrm{C}\left(\mathrm{CH}_{3}\right)_{3}\right), 51.5\left(\mathrm{OCH}_{3}\right), 51.6\left(\mathrm{PhCH}_{2}\right)$, $56.9\left(\mathrm{CH}_{2}\right), 71.1(\mathrm{C}-1), 127.6,128.1\left(\mathrm{C}-4^{\prime}, \mathrm{C}-4^{\prime \prime}\right), 128.5$, $128.6\left(\mathrm{C}-2^{\prime}, \mathrm{C}-6^{\prime}, \mathrm{C}-2^{\prime \prime}, \mathrm{C}-6^{\prime \prime}\right), 128.9,129.6\left(\mathrm{C}-3^{\prime}, \mathrm{C}-5^{\prime}, \mathrm{C}-3^{\prime \prime}\right.$, $\left.\mathrm{C}-5^{\prime \prime}\right), 135.6,137.8$ (C-1' $\left.{ }^{\prime}, \mathrm{C}-1^{\prime \prime}\right), 170.5$ (CONH), 172.1 $\left(\mathrm{COOCH}_{3}\right)$; HRMS (ESI+) calcd for $\mathrm{C}_{22} \mathrm{H}_{28} \mathrm{~N}_{2} \mathrm{O}_{3} \mathrm{Na}$ : $391.1998(\mathrm{M}+\mathrm{Na})^{+}$found 391.1985.

Synthesis of compounds 2 by $\mathrm{BF}_{3} \cdot 2 \mathrm{CH}_{3} \mathrm{COOH}$ mediated N-detertbutylation

The appropriate Ugi product $\mathbf{1}$ was dissolved in $\mathrm{BF}_{3} \cdot 2 \mathrm{CH}_{3} \mathrm{COOH}\left(\sim 36 \% \mathrm{BF}_{3}\right.$ basis, $3 \mathrm{~mL} / 1 \mathrm{mmol}$ of substrate), and stirred at $45-55{ }^{\circ} \mathrm{C}$ until full conversion of the starting material is observed by TLC (typically for 4-6 h). The solution was cooled, poured onto excess of crushed ice and made alkaline with $25 \%$ aqueous solution of ammonia. The resulting mixture was extracted with DCM $(3 \times 50 \mathrm{~mL})$. The combined organic phase was washed with water $(30 \mathrm{~mL})$, brine $(30 \mathrm{~mL})$, dried over anhydrous $\mathrm{MgSO}_{4}$, filtered and concentrated under reduced pressure. The residue was purified by FC.

Methyl (2S,1S)- and (2S,1S)-2-(2-amino-2-oxo-1phenylethylamino)-3-methylbutanoate $(2 S, 1 S)-2 a$ and $(2 S, 1 R)-2 a$

From diastereomeric mixture of $(\mathbf{2 S}, \mathbf{1 S})-\mathbf{1 a}$ and $(\mathbf{2 S}, \mathbf{1 R})-\mathbf{1 a}$ (3.98 g, $12.43 \mathrm{mmol})$ and $\mathrm{BF}_{3} \cdot 2 \mathrm{CH}_{3} \mathrm{COOH}(37 \mathrm{~mL})$; FC (gradient: PE/AcOEt 2:1-0:1): yield $2.31 \mathrm{~g}(70 \%): 1.95 \mathrm{~g}$ 
$(59 \%)$ of $(\mathbf{2 S , 1 S ) - 2 a}, 0.19 \mathrm{~g}(6 \%)$ of $(\mathbf{2 S}, \mathbf{1 R})-\mathbf{2 a}$ and $0.17 \mathrm{~g}(5 \%)$ of diastereomeric mixture. $(2 S, 1 S)-2 \mathrm{a}$ : colorless oil; $[\alpha]_{\mathrm{D}}=-133.5\left(c 0.977, \mathrm{CHCl}_{3}\right)$; IR $(\mathrm{KBr})$ : 702, 759, 1152, 1205, 1456, 1682, 1732, 2874, 2960, 3196, 3332, 3445; TLC (AcOEt): $R_{\mathrm{f}}=0.54 ;{ }^{1} \mathrm{H}$ NMR $\left(\mathrm{CDCl}_{3}\right.$, $500 \mathrm{MHz}): \delta 0.89\left(\mathrm{~d},{ }^{3} J=7.0,3 \mathrm{H}, \mathrm{CH}_{3}\right), 0.93(\mathrm{~d}$, $\left.{ }^{3} J=7.0,3 \mathrm{H}, \mathrm{CH}_{3}^{\prime}\right), 1.96\left(\mathrm{~m},{ }^{3} \mathrm{~J}=7.0,1 \mathrm{H}, \mathrm{CH}\right), 2.22(\mathrm{bs}$, $1 \mathrm{H}, \mathrm{NH}$ ), 2.87 (bs, $1 \mathrm{H}, \mathrm{H}-2), 3.72$ (s, 3H, $\mathrm{OCH}_{3}$ ), 4.19 (s, $1 \mathrm{H}, \mathrm{H}-1), 5.80$ (bs, $1 \mathrm{H}, \mathrm{CON} H$ ), 6.23 (bs, $1 \mathrm{H}, \mathrm{CONH}^{\prime}$ ), 7.30-7.40 (m, 5H, H-Ar); $\left.{ }^{13} \mathrm{C} \mathrm{NMR} \mathrm{(} \mathrm{CDCl}_{3}, 125 \mathrm{MHz}\right): \delta$ $18.4\left(\mathrm{CH}_{3}\right), 19.3\left(\mathrm{CH}_{3}^{\prime}\right), 31.4(\mathrm{CH}), 52.6\left(\mathrm{OCH}_{3}\right), 64.2(\mathrm{C}-$ 2), $65.6(\mathrm{C}-1), 128.1\left(\mathrm{C}-2^{\prime}, \mathrm{C}-6^{\prime}\right), 128.5\left(\mathrm{C}-4^{\prime}\right), 128.9\left(\mathrm{C}-3^{\prime}\right.$, $\left.\mathrm{C}-5^{\prime}\right), 138.1\left(\mathrm{C}-1^{\prime}\right), 174.3(\mathrm{CONH}), 174.8\left(\mathrm{COOCH}_{3}\right)$; HRMS (ESI) calcd for $\mathrm{C}_{14} \mathrm{H}_{20} \mathrm{~N}_{2} \mathrm{O}_{3} \mathrm{Na}$ : 287.1372 $(\mathrm{M}+\mathrm{Na})^{+}$found 287.1396. $(\mathbf{2 S , 1 R})-2 \mathrm{a}$ : white powder; $\mathrm{mp}$ $107-109{ }^{\circ} \mathrm{C} ;[\alpha]_{\mathrm{D}}=-5.2\left(\mathrm{c} 0.975, \mathrm{CHCl}_{3}\right)$; IR (KBr): 698, 758, 1150, 1202, 1456, 1685, 1733, 2874, 2960, 3196, 3331, 3443; TLC (AcOEt): $R_{\mathrm{f}}=0.58 ;{ }^{1} \mathrm{H} \mathrm{NMR}\left(\mathrm{CDCl}_{3}\right.$, $500 \mathrm{MHz}): \delta 0.96\left(\mathrm{~d},{ }^{3} J=7.0,3 \mathrm{H}, \mathrm{CH}_{3}\right), 1.03(\mathrm{~d}$, $\left.{ }^{3} J=7.0,3 \mathrm{H}, \mathrm{CH}_{3}^{\prime}\right), 2.02\left(\mathrm{~m},{ }^{3} J=7.0,1 \mathrm{H}, \mathrm{CH}\right), 2.18$ (bs, $1 \mathrm{H}, \mathrm{NH}$ ), 3.17 (bs, $1 \mathrm{H}, \mathrm{H}-2), 3.72$ (s, 3H, $\mathrm{OCH}_{3}$ ), 4.06 (s, $1 \mathrm{H}, \mathrm{H}-1), 5.93$ (bs, $1 \mathrm{H}, \mathrm{CONH}$ ), 7.22 (bs, $1 \mathrm{H}, \mathrm{CONH}^{\prime}$ ), 7.28-7.44 (m, 5H, H-Ar); $\left.{ }^{13} \mathrm{C} \mathrm{NMR} \mathrm{(} \mathrm{CDCl}_{3}, 125 \mathrm{MHz}\right): \delta$ $18.2\left(\mathrm{CH}_{3}\right), 19.6\left(\mathrm{CH}_{3}^{\prime}\right), 31.6(\mathrm{CH}), 51.8\left(\mathrm{OCH}_{3}\right), 66.2(\mathrm{C}-$ 1), 66.7 (C-2), 127.3 (C-2', C-6'), $128.4\left(\mathrm{C}-4^{\prime}\right), 128.9$ (C-3', $\left.\mathrm{C}-5^{\prime}\right), 138.8\left(\mathrm{C}-1^{\prime}\right), 174.8(\mathrm{CONH}), 174.9\left(\mathrm{COOCH}_{3}\right)$; HRMS (ESI) calcd for $\mathrm{C}_{14} \mathrm{H}_{20} \mathrm{~N}_{2} \mathrm{O}_{3} \mathrm{Na}$ : 287.1372 $(\mathrm{M}+\mathrm{Na})^{+}$found 287.1359 .

\section{Methyl (2S,1R)- and (2S,1S)-2-(2-amino-2-oxo-1- phenylethylamino)-4-methylpentanoate $(2 S, 1 S)-2 b$ and $(2 S, 1 R)-2 b$}

From diastereomeric mixture of $(\mathbf{2} S, \mathbf{1 S})$-1b and $(\mathbf{2} S, \mathbf{1 R})$-1b $(3.11 \mathrm{~g}, 9.31 \mathrm{mmol})$ and $\mathrm{BF}_{3} \cdot 2 \mathrm{CH}_{3} \mathrm{COOH}(28 \mathrm{~mL})$; $\mathrm{FC}$ (gradient: PE/AcOEt 2:1-0:1): yield $1.43 \mathrm{~g} \mathrm{(55 \% ):} 1.03 \mathrm{~g}$ $(40 \%)$ of $(\mathbf{2 S}, \mathbf{1 S})-\mathbf{2 b}, 0.08 \mathrm{~g}(3 \%)$ of $(\mathbf{2 S}, \mathbf{1 R})-\mathbf{2 b}$ and $0.32 \mathrm{~g}(12 \%)$ of diastereomeric mixture. $(\mathbf{2 S}, \mathbf{1 S})-\mathbf{2 b}$ : yellow wax; mp $65-72{ }^{\circ} \mathrm{C} ;[\alpha]_{\mathrm{D}}=-132.9\left(\right.$ c 1.107, $\left.\mathrm{CHCl}_{3}\right)$; IR (KBr): 702, 739, 1155, 1202, 1271, 1367, 1454, 1676, 1732, 2872, 2957, 3192, 3329, 3441; TLC (AcOEt): $R_{\mathrm{f}}=0.51 ;{ }^{1} \mathrm{H} \quad \mathrm{NMR}\left(\mathrm{CDCl}_{3}, 500 \mathrm{MHz}\right): \delta 0.73(\mathrm{~d}$, $\left.{ }^{3} J=6.5,3 \mathrm{H}, \mathrm{CH}_{3}\right), 0.87\left(\mathrm{~d},{ }^{3} J=6.5,3 \mathrm{H}, \mathrm{CH}_{3}^{\prime}\right), 1.47(\mathrm{~m}$, $\left.2 \mathrm{H}, \mathrm{CH}_{2}\right), 1.76\left(\mathrm{~m},{ }^{3} J_{1}=7.5,{ }^{3} J_{1}=6.5,1 \mathrm{H}, \mathrm{CH}\right), 2.44$ (bs, $1 \mathrm{H}, \mathrm{N} H), 3.11\left(\mathrm{dd},{ }^{3} J_{1}=8.5,{ }^{3} J_{1}=6.0,1 \mathrm{H}, \mathrm{H}-2\right)$, $3.70\left(\mathrm{~s}, 3 \mathrm{H}, \mathrm{OCH}_{3}\right), 4.24(\mathrm{~s}, 1 \mathrm{H}, \mathrm{H}-1), 5.93$ (bs, $1 \mathrm{H}$, $\mathrm{CONH}$ ), 6.31 (bs, 1H, CONH'), 7.29-7.39 (m, 5H, H-Ar); ${ }^{13} \mathrm{C}$ NMR $\left(\mathrm{CDCl}_{3}, 125 \mathrm{MHz}\right): \delta 21.8\left(\mathrm{CH}_{3}\right), 22.9\left(\mathrm{CH}_{3}^{\prime}\right)$, $24.7(\mathrm{CH}), 42.6\left(\mathrm{CH}_{2}\right), 51.8\left(\mathrm{OCH}_{3}\right), 57.0(\mathrm{C}-2), 65.3(\mathrm{C}-$ 1), $128.0\left(\mathrm{C}-2^{\prime}, \mathrm{C}-6^{\prime}\right), 128.5\left(\mathrm{C}-4^{\prime}\right), 128.9\left(\mathrm{C}-3^{\prime}, \mathrm{C}-5^{\prime}\right)$, $138.2\left(\mathrm{C}-1^{\prime}\right), 174.4(\mathrm{CONH}), 175.5\left(\mathrm{COOCH}_{3}\right)$; HRMS (ESI) calcd for $\mathrm{C}_{15} \mathrm{H}_{22} \mathrm{~N}_{2} \mathrm{O}_{3} \mathrm{Na}$ : $301.1528(\mathrm{M}+\mathrm{Na})^{+}$found 301.1522; (2S,1R)-2b: pale-yellow powder; $\mathrm{mp} 88-95{ }^{\circ} \mathrm{C}$;
$[\alpha]_{\mathrm{D}}=-0.2\left(c 1.030, \mathrm{CHCl}_{3}\right) ; \mathrm{IR}(\mathrm{KBr}): 702,756,1157$, $1202,1269,1387,1454,1680,1734,2870,2957,3190$, 3325,3445 ; TLC (AcOEt): $R_{\mathrm{f}}=0.63 ;{ }^{1} \mathrm{H}$ NMR $\left(\mathrm{CDCl}_{3}\right.$, $500 \mathrm{MHz}): \delta 0.95\left(\mathrm{~d},{ }^{3} J=6.5,3 \mathrm{H}, \mathrm{CH}_{3}\right), 0.95(\mathrm{~d}$, $\left.{ }^{3} J=6.5,3 \mathrm{H}, \mathrm{CH}_{3}^{\prime}\right), 1.49\left(\mathrm{~m}, 2 \mathrm{H}, \mathrm{CH}_{2}\right), 1.83\left(\mathrm{~m},{ }^{3} J=6.5\right.$, $1 \mathrm{H}, \mathrm{CH}), 2.25(\mathrm{bs}, 1 \mathrm{H}, \mathrm{NH}), 3.40\left(\mathrm{dd},{ }^{3} J_{1}=8.0,{ }^{3} J_{1}=6.0\right.$, $1 \mathrm{H}, \mathrm{H}-2), 3.70$ (s, 3H, OCH $\mathrm{OCH}_{3}, 4.10(\mathrm{~s}, 1 \mathrm{H}, \mathrm{H}-1), 6.08$ (bs, $1 \mathrm{H}, \mathrm{CONH}), 7.17\left(\mathrm{bs}, 1 \mathrm{H}, \mathrm{CON} H^{\prime}\right), 7.27-7.42(\mathrm{~m}, 5 \mathrm{H}, \mathrm{H}-$ $\mathrm{Ar}) ;{ }^{13} \mathrm{C}$ NMR $\left(\mathrm{CDCl}_{3}, 125 \mathrm{MHz}\right): \delta 22.0\left(\mathrm{CH}_{3}\right), 22.9$ $\left(\mathrm{CH}_{3}^{\prime}\right), 24.9(\mathrm{CH}), 43.1\left(\mathrm{CH}_{2}\right), 51.9\left(\mathrm{OCH}_{3}\right), 59.0(\mathrm{C}-2)$, $66.3(\mathrm{C}-1), 127.2\left(\mathrm{C}-2^{\prime}, \mathrm{C}^{\prime} 6^{\prime}\right), 128.3\left(\mathrm{C}-4^{\prime}\right), 128.8\left(\mathrm{C}-3^{\prime}\right.$, $\left.\mathrm{C}-5^{\prime}\right), 138.7\left(\mathrm{C}-1^{\prime}\right), 175.0(\mathrm{CONH}), 175.7\left(\mathrm{COOCH}_{3}\right)$; HRMS (ESI) calcd for $\mathrm{C}_{15} \mathrm{H}_{22} \mathrm{~N}_{2} \mathrm{O}_{3} \mathrm{Na}$ : 301.1528 $(\mathrm{M}+\mathrm{Na})^{+}$found 301.1534 .

Methyl (2S, 1R,3S)- and (2S,1S,3S)-2-(2-amino-2-oxo-1phenylethylamino)-3-methylpentanoate (2S,1R,3S)-2c and $(2 S, 1 S, 3 S)-2 c$

From diastereomeric mixture of $(2 S, 1 S, 3 S)-1 c$ and $(2 S, 1 R, 3 S)-1 \mathrm{c}(3.96 \mathrm{~g}, 11.85 \mathrm{mmol})$ and $\mathrm{BF}_{3} \cdot 2 \mathrm{CH}_{3} \mathrm{COOH}$ $(35 \mathrm{~mL}$ ); FC (gradient: PE/AcOEt 2:1-0:1): yield $2.75 \mathrm{~g}$ (83\%): $1.92 \mathrm{~g}(58 \%)$ of $(\mathbf{2 S , 1 S , 3 S ) - 2 c , ~} 0.05 \mathrm{~g}(1 \%)$ of $(2 S, 1 R, 3 S)-1 \mathrm{c}$ and $0.78 \mathrm{~g}(24 \%)$ of diastereomeric mixture. $(\mathbf{2 S}, \mathbf{1 S}, \mathbf{3 S})-2 \mathrm{c}$ : pale-yellow oil; $[\alpha]_{\mathrm{D}}=-124.1(c$ $0.085, \mathrm{CHCl}_{3}$ ); IR (KBr): 702, 758, 1151, 1202, 1384, 1456, 1682, 1734, 2878, 2964, 3190, 3325, 3447; TLC $(\mathrm{AcOEt}): R_{\mathrm{f}}=0.55 ;{ }^{1} \mathrm{H}$ NMR $\left(\mathrm{CDCl}_{3}, 500 \mathrm{MHz}\right): \delta 0.83$ $\left(\mathrm{t},{ }^{3} \mathrm{~J}=7.5,3 \mathrm{H}, \mathrm{CH}_{2} \mathrm{CH}_{3}\right), 0.85\left(\mathrm{~d},{ }^{3} \mathrm{~J}=7.0,3 \mathrm{H}, \mathrm{CH}_{3}\right)$, $1.17\left(\mathrm{~m}, 1 \mathrm{H}, \mathrm{CH}_{2}\right), 1.52\left(\mathrm{~m}, 1 \mathrm{H}, \mathrm{CH}_{2}^{\prime}\right), 1.71(\mathrm{~m}, 1 \mathrm{H}, \mathrm{CH})$, 2.54 (bs, $1 \mathrm{H}, \mathrm{NH}), 2.94\left(\mathrm{~d},{ }^{3} \mathrm{~J}=6.0,1 \mathrm{H}, \mathrm{H}-2\right), 3.71(\mathrm{~s}, 3 \mathrm{H}$, $\left.\mathrm{OCH}_{3}\right), 4.19$ (s, $\left.1 \mathrm{H}, \mathrm{H}-1\right), 5.73$ (bs, $\left.1 \mathrm{H}, \mathrm{CON} H^{\prime}\right), 6.23$ (bs, $1 \mathrm{H}, \mathrm{CONH}), 7.31-7.42$ (m, 5H, H-Ar); ${ }^{13} \mathrm{C} \mathrm{NMR}\left(\mathrm{CDCl}_{3}\right.$, $125 \mathrm{MHz}): \delta 11.3,15.6\left(\mathrm{CH}_{3}, \mathrm{CH}_{3}^{\prime}\right), 25.2\left(\mathrm{CH}_{2}\right), 38.0$ $(\mathrm{CH}), 51.6\left(\mathrm{OCH}_{3}\right), 63.2(\mathrm{C}-2), 65.6(\mathrm{C}-1), 128.1\left(\mathrm{C}-2^{\prime}, \mathrm{C}-\right.$ $\left.6^{\prime}\right), 128.5\left(\mathrm{C}-4^{\prime}\right), 128.9\left(\mathrm{C}-3^{\prime}, \mathrm{C}-5^{\prime}\right), 138.1\left(\mathrm{C}-1^{\prime}\right), 174.3$ $(\mathrm{CONH}), 174.8\left(\mathrm{COOCH}_{3}\right)$; HRMS (ESI) calcd for $\mathrm{C}_{15} \mathrm{H}_{22} \mathrm{~N}_{2} \mathrm{O}_{3} \mathrm{Na}$ : $301.1528(\mathrm{M}+\mathrm{Na})^{+}$found 301.1516;

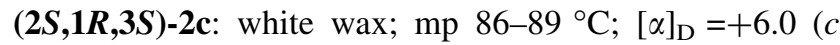
$0.833, \mathrm{CHCl}_{3}$ ); IR (KBr): 700, 756, 1150, 1202, 1267, 1381, 1456, 1680, 1732, 2878, 2964, 3194, 3331, 3443; TLC (AcOEt): $R_{\mathrm{f}}=0.63 ;{ }^{1} \mathrm{H}$ NMR $\left(\mathrm{CDCl}_{3}, 500 \mathrm{MHz}\right): \delta$ $0.91\left(\mathrm{t},{ }^{3} \mathrm{~J}=7.5,3 \mathrm{H}, \mathrm{CH}_{2} \mathrm{CH}_{3}\right), 0.97\left(\mathrm{~d},{ }^{3} \mathrm{~J}=7.0,3 \mathrm{H}\right.$, $\left.\mathrm{CH}_{3}\right), 1.20\left(\mathrm{~m}, 1 \mathrm{H}, \mathrm{CH}_{2}\right), 1.54\left(\mathrm{~m}, 1 \mathrm{H}, \mathrm{CH}_{2}^{\prime}\right), 1.76(\mathrm{~m}, 1 \mathrm{H}$, $\mathrm{CH}), 2.22(\mathrm{bs}, 1 \mathrm{H}, \mathrm{NH}), 3.25\left(\mathrm{~d},{ }^{3} J=5.5,1 \mathrm{H}, \mathrm{H}-2\right), 3.71$ (s, 3H, $\mathrm{OCH}_{3}$ ), 4.06 (s, $\left.1 \mathrm{H}, \mathrm{H}-1\right), 6.06$ (bs, $1 \mathrm{H}, \mathrm{CON} H^{\prime}$ ), 7.20 (bs, $1 \mathrm{H}, \mathrm{CON} H$ ), 7.28-7.42 (m, 5H, H-Ar); ${ }^{13} \mathrm{C}$ NMR $\left(\mathrm{CDCl}_{3}, 125 \mathrm{MHz}\right): \delta 11.6,15.9\left(\mathrm{CH}_{3}, \mathrm{CH}_{3}^{\prime}\right), 25.2\left(\mathrm{CH}_{2}\right)$, $38.5(\mathrm{CH}), 51.7\left(\mathrm{OCH}_{3}\right), 65.3(\mathrm{C}-2), 66.7(\mathrm{C}-1), 127.3(\mathrm{C}-$ $\left.2^{\prime}, \mathrm{C}-6^{\prime}\right), 128.3\left(\mathrm{C}-4^{\prime}\right), 128.9\left(\mathrm{C}-3^{\prime}, \mathrm{C}-5^{\prime}\right), 138.8\left(\mathrm{C}-1^{\prime}\right)$, $174.8(\mathrm{CONH}), 175.0\left(\mathrm{COOCH}_{3}\right)$; HRMS (ESI) calcd for $\mathrm{C}_{15} \mathrm{H}_{22} \mathrm{~N}_{2} \mathrm{O}_{3} \mathrm{Na}$ : $301.1528(\mathrm{M}+\mathrm{Na})^{+}$found 301.1501. 
Methyl (2S,1R)- and (2S,1S)-2-(2-amino-2-oxo-1phenylethylamino)-3-phenylpropanoate $(2 S, 1 R)-2 d$ and $(2 S, 1 S)-2 d$

From diastereomeric mixture of $(\mathbf{2 S}, \mathbf{1 S})-\mathbf{1 d}$ and $(\mathbf{2 S}, \mathbf{1 R})-\mathbf{1 d}$ $(2.34 \mathrm{~g}, 6.36 \mathrm{mmol})$ and $\mathrm{BF}_{3} \cdot 2 \mathrm{CH}_{3} \mathrm{COOH}(19 \mathrm{~mL}) ; \mathrm{FC}$ (gradient: PE/AcOEt 2:1-0:1): yield $1.32 \mathrm{~g}(67 \%): 1.10 \mathrm{~g}$ $(55 \%)$ of $(\mathbf{2 S}, \mathbf{1 S})-2 \mathrm{~d}$ and $0.22 \mathrm{~g}(12 \%)$ of $(\mathbf{2 S}, \mathbf{1 R})-\mathbf{2 d}$. (2S,1S)-2d: pale-yellow oil; $[\alpha]_{\mathrm{D}}=-72.3$ (c 0.392 , $\mathrm{CHCl}_{3}$ ); IR (KBr): 702, 752, 1205, 1454, 1682, 1734, 2854, 2951, 3028, 3190, 3325, 3445; TLC (AcOEt): $R_{\mathrm{f}}=0.46$; ${ }^{1} \mathrm{H}$ NMR $\left(\mathrm{CDCl}_{3}, 500 \mathrm{MHz}\right): \delta 2.40(\mathrm{bs}, 1 \mathrm{H}, \mathrm{N} H), 2.85$ $\left(\mathrm{dd},{ }^{2} J=13.5,{ }^{3} J=8.0,1 \mathrm{H}, \mathrm{CH}_{2}\right), 3.03\left(\mathrm{dd},{ }^{2} J=13.5\right.$, $\left.{ }^{3} J=6.0,1 \mathrm{H}, \mathrm{CH}_{2}^{\prime}\right), 3.38\left(\mathrm{bpt},{ }^{3} J=6.0,1 \mathrm{H}, \mathrm{H}-2\right), 3.67(\mathrm{~s}$, $3 \mathrm{H}, \mathrm{OCH}_{3}$ ), 4.22 (s, 1H, H-1), 5.60 (bs, 1H, CONH), 6.44 (bs, 1H, CONH $H^{\prime}$, 7.09 (m, 2H, H-Ar), 7.12 (m, 2H, H-Ar), 7.21-7.30 (m, 6H, H-Ar); ${ }^{13} \mathrm{C} \mathrm{NMR}\left(\mathrm{CDCl}_{3}, 125 \mathrm{MHz}\right): \delta$ $39.4\left(\mathrm{CH}_{2}\right), 51.9\left(\mathrm{OCH}_{3}\right), 60.1(\mathrm{C}-2), 65.3(\mathrm{C}-1), 126.8(\mathrm{C}-$ $\left.4^{\prime \prime}\right), 127.7\left(\mathrm{C}-2^{\prime}, \mathrm{C}-6^{\prime}\right), 128.3\left(\mathrm{C}-4^{\prime}\right), 128.4\left(\mathrm{C}-2^{\prime \prime}, \mathrm{C}-6^{\prime \prime}\right)$, $128.8\left(\mathrm{C}-3^{\prime}, \mathrm{C}-5^{\prime}\right), 129.2\left(\mathrm{C}-3^{\prime \prime}, \mathrm{C}-5^{\prime \prime}\right), 136.9\left(\mathrm{C}-1^{\prime \prime}\right), 137.7$ $\left(\mathrm{C}-1^{\prime}\right), 174.1\left(\mathrm{COOCH}_{3}\right), 174.2(\mathrm{CONH})$; HRMS (ESI) calcd for $\mathrm{C}_{18} \mathrm{H}_{20} \mathrm{~N}_{2} \mathrm{O}_{3} \mathrm{Na}$ : $335.1372(\mathrm{M}+\mathrm{Na})^{+}$found 335.1363. (2S,1R)-2d: white powder; mp $124-127^{\circ} \mathrm{C}$; $[\alpha]_{\mathrm{D}}=-37.8\left(c 0.775, \mathrm{CHCl}_{3}\right)$; IR $(\mathrm{KBr}): 702,739,1209$, 1452, 1693, 1734, 2951, 3030, 3188, 3335, 3429; TLC $(\mathrm{AcOEt}): R_{\mathrm{f}}=0.58 ;{ }^{1} \mathrm{H} \mathrm{NMR}\left(\mathrm{CDCl}_{3}, 500 \mathrm{MHz}\right): \delta 2.21$ (bs, $1 \mathrm{H}, \mathrm{NH}), 2.68\left(\mathrm{dd},{ }^{2} J=13.5,{ }^{3} J=10.0,1 \mathrm{H}, \mathrm{CH}_{2}\right.$ ), $3.11\left(\mathrm{dd},{ }^{2} \mathrm{~J}=13.5,{ }^{3} \mathrm{~J}=4.0,1 \mathrm{H}, \mathrm{CH}_{2}^{\prime}\right), 3.47 \quad(\mathrm{bps}$, $\left.{ }^{3} J=6.0,1 \mathrm{H}, \mathrm{H}-2\right), 3.76$ (s, 3H, OCH $\mathrm{OC}_{3}, 4.08$ (s, 1H, H-1), 5.04 (bs, $1 \mathrm{H}, \mathrm{CONH}$ ), 6.32 (bs, $1 \mathrm{H}, \mathrm{CON}^{\prime}$ ), 7.23-7.42 $(\mathrm{m}, 10 \mathrm{H}, \mathrm{H}-\mathrm{Ar}) ;{ }^{13} \mathrm{C} \mathrm{NMR}\left(\mathrm{CDCl}_{3}, 125 \mathrm{MHz}\right): \delta 40.1$ $\left(\mathrm{CH}_{2}\right), 52.2\left(\mathrm{OCH}_{3}\right), 62.3(\mathrm{C}-2), 66.4(\mathrm{C}-1), 127.0\left(\mathrm{C}-4^{\prime \prime}\right)$, $127.3\left(\mathrm{C}-2^{\prime}, \mathrm{C}-6^{\prime}\right), 128.4\left(\mathrm{C}-4^{\prime}\right), 128.6\left(\mathrm{C}-2^{\prime \prime}, \mathrm{C}-6^{\prime \prime}\right), 128.9$ $\left(\mathrm{C}-3^{\prime}, \mathrm{C}-5^{\prime}\right), 129.6\left(\mathrm{C}-3^{\prime \prime}, \mathrm{C}-5^{\prime \prime}\right), 137.7\left(\mathrm{C}-1^{\prime \prime}\right), 138.6\left(\mathrm{C}-1^{\prime}\right)$, $174.5\left(\mathrm{COOCH}_{3}\right), \quad 174.6 \quad(\mathrm{CONH}) ; \quad \mathrm{C}_{18} \mathrm{H}_{20} \mathrm{~N}_{2} \mathrm{O}_{3} \mathrm{Na}$ : $335.1372(\mathrm{M}+\mathrm{Na})^{+}$found 335.1366 .

\section{Methyl (2S,1S)- and (2S,1R)-2-(2-amino-2-oxo-1- phenylethylamino)-3-phenylacetate $(2 S, 1 S)-2 e$ and $(2 S, 1 R)-2 e$}

From diastereomeric mixture of $(\mathbf{2 S}, \mathbf{1 S})-\mathbf{1 e}$ and $(\mathbf{2 S}, \mathbf{1 R})-\mathbf{1 e}$ $(2.26 \mathrm{~g}, 6.38 \mathrm{mmol})$ and $\mathrm{BF}_{3} \cdot 2 \mathrm{CH}_{3} \mathrm{COOH}(19 \mathrm{~mL}) ; \mathrm{FC}$ (gradient: PE/AcOEt 4:1-1:2): yield $1.54 \mathrm{~g}(81 \%)$ of diastereomeric mixture $\left(d_{\mathrm{r}}=1.4 / 1,{ }^{1} \mathrm{H}\right.$ NMR). Pale-yellow, sticky wax; mp 43-50 ${ }^{\circ} \mathrm{C}$; IR (KBr): 700, 741, 1175, 1209, 1456, 1510, 1558, 1682, 1736, 2852, 2924, 3194, 3333, 3443; TLC (AcOEt): $R_{\mathrm{f}}=0.51 ;{ }^{1} \mathrm{H} \mathrm{NMR}$ (from diastereomeric mixture, $\left.\mathrm{CDCl}_{3}, 500 \mathrm{MHz}\right)$ : $(\mathbf{2 S , 1 S ) - 2 e}$ (major isomer): $\delta 2.85(\mathrm{bs}, 1 \mathrm{H}, \mathrm{NH}), 3.69\left(\mathrm{~s}, 3 \mathrm{H}, \mathrm{OCH}_{3}\right.$ ), 4.13 (s, 1H, H-1), 4.39 (s, 1H, H-2), 5.29 (bs, 1H, CONH), 6.83 (bs, 1H, CONH $\left.H^{\prime}\right), 7.28-7.40$ (m, 10H, H-Ar); (2S,1R)-2e (minor isomer): $\delta 2.98$ (bs, 1H, NH), 3.68 (s, 3H, $\left.\mathrm{OCH}_{3}\right), 4.33$ (s, 1H, H-2), 5.29 (bs, 1H, CONH), $6.80\left(\mathrm{bs}, 1 \mathrm{H}, \mathrm{CON} H^{\prime}\right)$; the remaining signals overlap with the signals of $(\mathbf{2 S}, \mathbf{1 S})-\mathbf{2} \mathrm{e} ;{ }^{13} \mathrm{C} \mathrm{NMR}$ (from diastereomeric mixture, $\left.\mathrm{CDCl}_{3}, 125 \mathrm{MHz}\right)$ : $(\mathbf{2 S}, \mathbf{1 S})-\mathbf{2 e}$ (major isomer): $\delta$ $52.4\left(\mathrm{OCH}_{3}\right), 63.3(\mathrm{C}-2), 64.3(\mathrm{C}-1), 127.6\left(\mathrm{C}-2^{\prime}, \mathrm{C}-6^{\prime}\right.$, $\left.\mathrm{C}-2^{\prime \prime}, \mathrm{C}-6^{\prime \prime}\right), 128.5,128.6\left(\mathrm{C}-4^{\prime}, \mathrm{C}-4^{\prime \prime}\right), 129.0\left(\mathrm{C}-3^{\prime}, \mathrm{C}-5^{\prime}\right.$, $\left.\mathrm{C}-3^{\prime \prime}, \mathrm{C}-5^{\prime \prime}\right), 137.1,138.3\left(\mathrm{C}-1^{\prime}, \mathrm{C}-1^{\prime \prime}\right), 172.6$ (CONH), $174.3\left(\mathrm{COOCH}_{3}\right) ;(\mathbf{2 S , 1 R})-2 \mathrm{e}$ (minor isomer): $\delta 52.5$ $\left(\mathrm{OCH}_{3}\right), 63.5(\mathrm{C}-2), 65.3(\mathrm{C}-1), 127.4,127.7\left(\mathrm{C}-2^{\prime}, \mathrm{C}-6^{\prime}\right.$, $\left.\mathrm{C}-2^{\prime \prime}, \mathrm{C}-6^{\prime \prime}\right), 128.4,128.5\left(\mathrm{C}-4^{\prime}, \mathrm{C}-4^{\prime \prime}\right), 128.9,129.0\left(\mathrm{C}-3^{\prime}\right.$, $\left.\mathrm{C}-5^{\prime}, \mathrm{C}-3^{\prime \prime}, \mathrm{C}-5^{\prime \prime}\right), \quad 137.3,138.1 \quad\left(\mathrm{C}-1^{\prime}, \mathrm{C}-1^{\prime \prime}\right), 173.0$ $(\mathrm{CONH}), 174.4\left(\mathrm{COOCH}_{3}\right)$; HRMS (ESI+) calcd for $\mathrm{C}_{17} \mathrm{H}_{18} \mathrm{~N}_{2} \mathrm{O}_{3} \mathrm{Na}$ : $321.1215(\mathrm{M}+\mathrm{Na})^{+}$found 321.1227.

Methyl (+/-)-2-(2-benzyl-2-amino-2-oxo-1phenylethylamino)-acetate rac- $2 f$

From rac-1f $(0.59 \mathrm{~g}, 1.60 \mathrm{mmol})$ and $\mathrm{BF}_{3} \cdot 2 \mathrm{CH}_{3} \mathrm{COOH}$ (5 mL); FC (gradient: PE/AcOEt 4:1-1:2): yield $0.40 \mathrm{~g}$ $(80 \%)$ of rac-2f. White powder; mp 147-149 ${ }^{\circ} \mathrm{C}$; TLC (AcOEt): $R_{\mathrm{f}}=0.63$; IR (KBr): 700, 741, 1204, 1454, 1558, 1682, 1734, 2844, 2951, 3030, 3182, 3418; ${ }^{1} \mathrm{H}$ NMR $\left(\mathrm{CDCl}_{3}, 500 \mathrm{MHz}\right): \delta 3.07\left(\mathrm{~d},{ }^{2} J=17.5,1 \mathrm{H}, \mathrm{PhCH}_{2}\right)$, $3.40\left(\mathrm{~d},{ }^{2} \mathrm{~J}=17.5,1 \mathrm{H}, \mathrm{PhCH}_{2}^{\prime}\right), 3.61\left(\mathrm{~s}, 3 \mathrm{H}, \mathrm{OCH}_{3}\right), 3.66$ $\left(\mathrm{d},{ }^{2} J=13.5,1 \mathrm{H}, \mathrm{CH}_{2}\right), 3.85\left(\mathrm{~d},{ }^{2} \mathrm{~J}=13.5,1 \mathrm{H}, \mathrm{CH}_{2}^{\prime}\right)$, 4.75 (s, 1H, H-1), 5.85 (bs, 1H, CONH), 7.26-7.42 (m, $10 \mathrm{H}, \mathrm{H}-\mathrm{Ar}), 7.63$ (bs, $\left.1 \mathrm{H}, \mathrm{CON} H^{\prime}\right) ;{ }^{13} \mathrm{C} \mathrm{NMR}\left(\mathrm{CDCl}_{3}\right.$, $125 \mathrm{MHz}): \delta 51.7\left(\mathrm{OCH}_{3}\right), 51.8\left(\mathrm{PhCH}_{2}\right), 56.8\left(\mathrm{CH}_{2}\right), 69.9$ (C-1), 127.7, 128.4 (C-4', C-4"), 128.64, 128.65 (C-2', C-6', C-2" ${ }^{\prime \prime}$, C-6" $\left.{ }^{\prime \prime}\right), 129.0,129.6\left(\mathrm{C}-3^{\prime}, \mathrm{C}-5^{\prime}, \mathrm{C}-3^{\prime \prime}, \mathrm{C}-5^{\prime \prime}\right)$,

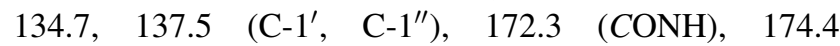
$\left(\mathrm{COOCH}_{3}\right)$; HRMS (ESI+) calcd for $\mathrm{C}_{18} \mathrm{H}_{20} \mathrm{~N}_{2} \mathrm{O}_{3} \mathrm{Na}$ : $335.1360(\mathrm{M}+\mathrm{Na})^{+}$found 335.1372.

Synthesis of compounds 3 by base-induced intramolecular cyclocondensation

To a stirred solution of appropriate amidoester $\mathbf{2}$ in absolute $\mathrm{EtOH}$ (5 mL/1 mmol of amidoester), sodium hydroxide (1 equiv.) was added at room temperature. After dissolution of the hydroxide, the mixture was quenched with saturated aqueous solution of ammonium chloride (100 $\mathrm{mL})$. The resulting cloudy solution was extracted with $\mathrm{CH}_{2} \mathrm{Cl}_{2}(3 \times 30 \mathrm{~mL})$. The combined organic phase was washed with water $(20 \mathrm{~mL})$, dried over anhydrous $\mathrm{MgSO}_{4}$, filtered and concentrated under reduced pressure. The residue was purified by $\mathrm{FC}$.

(3S,5R)- and (3S,5S)-3-isopropyl-5-phenylpiperazine-2,6dione $(3 S, 5 S)-3 a$ and $(3 S, 5 R)-3 a$

From (2S,1S)-2a (1.86 g, $7.04 \mathrm{mmol})$ and $\mathrm{NaOH}(0.28 \mathrm{~g}$, 1 equiv.); FC (gradient: PE/EtOAc 6:1-1:1): yield $1.34 \mathrm{~g}$ 
(82\%): $0.72 \mathrm{~g}(44 \%)$ of $(\mathbf{3 S}, \mathbf{5 S})-3 \mathrm{a}, 0.32 \mathrm{~g}(19 \%)$ of $(\mathbf{3 S}, \mathbf{5 R})-3 \mathrm{a}$ and $0.30 \mathrm{~g}(19 \%)$ of diastereomeric mixture. (3S,5S)-3a: white powder; $m p$ 103-105 ${ }^{\circ} \mathrm{C} ;[\alpha]_{\mathrm{D}}=-152.1$ (c 1, $\mathrm{CHCl}_{3}$ ); IR (KBr): 756, 1030, 1099, 1180, 1234, 1331, 1454, 1497, 1701, 2932, 1963, 3225; TLC (PE/AcOEt 3:1): $R_{\mathrm{f}}=0.35 ;{ }^{1} \mathrm{H} \quad \mathrm{NMR} \quad\left(\mathrm{CDCl}_{3}, 500 \mathrm{MHz}\right): \delta 0.99 \quad(\mathrm{~d}$, $\left.{ }^{3} J=7.0,3 \mathrm{H}, \mathrm{CH}_{3}\right), 1.09\left(\mathrm{~d},{ }^{3} J=7.0,3 \mathrm{H}, \mathrm{CH}_{3}^{\prime}\right), 2.18(\mathrm{bs}$, $1 \mathrm{H}, \mathrm{N} H), 2.49\left(2 \mathrm{sp},{ }^{3} J_{1}=6.5,{ }^{3} J_{2}=5.0,1 \mathrm{H}, \mathrm{CH}\right), 3.26(\mathrm{~d}$, $\left.{ }^{3} J=4.5,1 \mathrm{H}, \mathrm{H}-3\right), 4.90(\mathrm{~s}, 1 \mathrm{H}, \mathrm{H}-5), 7.32-7.46(\mathrm{~m}, 5 \mathrm{H}, \mathrm{H}-$ Ar), 8.34 (bs, $1 \mathrm{H}, \mathrm{CONHCO}) ;{ }^{13} \mathrm{C} \mathrm{NMR} \quad\left(\mathrm{CDCl}_{3}\right.$, $125 \mathrm{MHz}): \delta 17.1\left(\mathrm{CH}_{3}\right), 19.3\left(\mathrm{CH}_{3}\right), 27.7(\mathrm{CH}), 58.7(\mathrm{C}-$ 3), 59.8 (C-5), 127.1 (C-2', C-6'), $128.5\left(\mathrm{C}-4^{\prime}\right), 129.0$ (C-3', C-5'), 134.6 (C-1' $), 172.3$ (C-6), 173.1 (C-2); HRMS (ESI+) calcd for $\mathrm{C}_{13} \mathrm{H}_{16} \mathrm{~N}_{2} \mathrm{O}_{2} \mathrm{Na}$ : $255.1109(\mathrm{M}+\mathrm{Na})^{+}$ found 255.1101. (3S,5R)-3a: white powder; mp $111-112{ }^{\circ} \mathrm{C} ;[\alpha]_{\mathrm{D}}=-117.5\left(c 1, \mathrm{CHCl}_{3}\right)$; IR (KBr): 756, 1223, 1269, 1497, 1701, 2874, 2936, 3032, 3221; TLC (PE/ AcOEt 3:1): $R_{\mathrm{f}}=0.29 ;{ }^{1} \mathrm{H} \mathrm{NMR}\left(\mathrm{CDCl}_{3}, 500 \mathrm{MHz}\right): \delta$ $1.02\left(\mathrm{~d},{ }^{3} \mathrm{~J}=7.0,3 \mathrm{H}, \mathrm{CH}_{3}\right), 1.09\left(\mathrm{~d},{ }^{3} \mathrm{~J}=7.0,3 \mathrm{H}, \mathrm{CH}_{3}^{\prime}\right)$, $1.76(\mathrm{bs}, 1 \mathrm{H}, \mathrm{NH}), 2.60\left(2 \mathrm{sp},{ }^{3} J_{1}=7.0,{ }^{3} J_{2}=2.5,1 \mathrm{H}\right.$, $\mathrm{CH}), 3.58\left(\mathrm{~d},{ }^{3} \mathrm{~J}=2.5,1 \mathrm{H}, \mathrm{H}-3\right), 4.54$ (s, 1H, H-5), 7.367.44 (m, 5H, H-Ar), 8.13 (bs, $1 \mathrm{H}, \mathrm{CONHCO}) ;{ }^{13} \mathrm{C} \mathrm{NMR}$ $\left(\mathrm{CDCl}_{3}, 125 \mathrm{MHz}\right): \delta 16.7\left(\mathrm{CH}_{3}\right), 19.3\left(\mathrm{CH}_{3}^{\prime}\right), 28.8(\mathrm{CH})$, 64.3 (C-3), 64.3 (C-5), 128.6 (C-2', C-6'), 128.8 (C-3', C-5') $128.9\left({\mathrm{C}-4^{\prime}}^{\prime}\right), 136.4\left(\mathrm{C}-1^{\prime}\right), 171.6$ (C-6), 172.3 (C-2); HRMS (ESI+) calcd for $\mathrm{C}_{13} \mathrm{H}_{16} \mathrm{~N}_{2} \mathrm{O}_{2} \mathrm{Na}$ : 255.1109 $(\mathrm{M}+\mathrm{Na})^{+}$found 255.1129 .

(3S,5S)- and (3S,5R)-3-isobutyl-5-phenylpiperazine-2,6dione (3S,5S)-3b and (3S,5R)-3b

From (2S,1S)-2b $(0.92 \mathrm{~g}, 3.31 \mathrm{mmol})$ and $\mathrm{NaOH}(0.13 \mathrm{~g}$, 1 equiv.); FC (gradient: PE/EtOAc 5:1-2:1): yield $0.63 \mathrm{~g}$ (77\%) of chromatographically inseparable diastereomeric mixture $\left(d_{\mathrm{r}}=68 / 32,{ }^{1} \mathrm{H}\right.$ NMR). Pure (3S,5S)-3b was obtained by fractional recrystallization form $\mathrm{PE} / \mathrm{Et}_{2} \mathrm{O} 1: 1$. (3S,5S)-3b: white powder; $m p$ 60-61 ${ }^{\circ} \mathrm{C} ;[\alpha]_{\mathrm{D}}=-30.3(c$ 1, $\mathrm{CHCl}_{3}$ ); IR (KBr): 756, 1242, 1384, 1454, 1701, 2870, 2955, 3090, 3225, 3321; TLC (PE/AcOEt 3:1): $R_{\mathrm{f}}=0.36$; ${ }^{1} \mathrm{H} \mathrm{NMR}\left(\mathrm{CDCl}_{3}, 500 \mathrm{MHz}\right): \delta 0.84\left(\mathrm{~d},{ }^{3} \mathrm{~J}=6.5,3 \mathrm{H}, \mathrm{CH}_{3}\right)$, $0.97\left(\mathrm{~d},{ }^{3} \mathrm{~J}=6.5,3 \mathrm{H}, \mathrm{CH}_{3}^{\prime}\right), 1.57\left(\mathrm{~m},{ }^{2} \mathrm{~J}=13.5,{ }^{3} J_{1}=9.5\right.$, $\left.{ }^{3} J_{2}=4.0,1 \mathrm{H}, \mathrm{CH}_{2}\right), 1.85\left(\mathrm{~m}, 1 \mathrm{H}, \mathrm{CH}_{2}^{\prime}\right), 1.89(\mathrm{~m}, 1 \mathrm{H}, \mathrm{CH})$, 2.07 (bs, $1 \mathrm{H}, \mathrm{NH}), 3.44$ (pd, $\left.{ }^{3} J=9.5,1 \mathrm{H}, \mathrm{H}-3\right), 4.86$ (s, 1H, H-5), 7.30-7.47 (m, 5H, H-Ar), 8.34 (bs, 1H, CONHCO); ${ }^{13} \mathrm{C} \mathrm{NMR}\left(\mathrm{CDCl}_{3}, 125 \mathrm{MHz}\right): \delta 21.1\left(\mathrm{CH}_{3}\right), 23.3\left(\mathrm{CH}_{3}^{\prime}\right)$, $24.4(\mathrm{CH}), 38.7\left(\mathrm{CH}_{2}\right), 52.1(\mathrm{C}-3), 59.7(\mathrm{C}-5), 127.2\left(\mathrm{C}-2^{\prime}\right.$, C-6 $\left.{ }^{\prime}\right), 128.5\left(\mathrm{C}-4^{\prime}\right), 128.9\left(\mathrm{C}-3^{\prime}, \mathrm{C}-5^{\prime}\right), 134.7\left(\mathrm{C}-1^{\prime}\right)$, 172.3 (C-6), 174.3 (C-2); HRMS (ESI+) calcd for $\mathrm{C}_{14} \mathrm{H}_{18} \mathrm{~N}_{2} \mathrm{O}_{2} \mathrm{Na}: 269.1266(\mathrm{M}+\mathrm{Na})^{+}$found 269.1231; (3S,5R)-3b: ${ }^{1} \mathrm{H}$ NMR (from diastereomeric mixture, $\mathrm{CDCl}_{3}$, $500 \mathrm{MHz}): \delta 0.95\left(\mathrm{~d},{ }^{3} \mathrm{~J}=6.5,3 \mathrm{H}, \mathrm{CH}_{3}\right), 0.98\left(\mathrm{~d},{ }^{3} \mathrm{~J}=6.5\right.$, $\left.3 \mathrm{H}, \mathrm{CH}_{3}^{\prime}\right), 1.61\left(\mathrm{~m}, 1 \mathrm{H}, \mathrm{CH}_{2}\right), 1.87(\mathrm{~m}, 2 \mathrm{H}, \mathrm{CH}, \mathrm{NH}), 2.02$ $\left(\mathrm{m},{ }^{2} J=14.0,{ }^{3} J_{1}=10.0,{ }^{3} J_{2}=4.0,1 \mathrm{H}, \mathrm{CH}_{2}^{\prime}\right), 3.66$ (m, 1H, H-3), 4.57 (s, 1H, H-5), 8.18 (bs, 1H, CONHCO), the remaining signals overlap with the signals of $(3 S, 5 S)-3 b$; ${ }^{13} \mathrm{C} \mathrm{NMR}$ (from diastereomeric mixture, $\mathrm{CDCl}_{3}, 125 \mathrm{MHz}$ ): $\delta 21.3\left(\mathrm{CH}_{3}\right), 23.4\left(\mathrm{CH}_{3}^{\prime}\right), 24.5(\mathrm{CH}), 39.0\left(\mathrm{CH}_{2}\right), 57.6(\mathrm{C}-3)$, $64.6(\mathrm{C}-5), 128.5\left(\mathrm{C}-2^{\prime}, \mathrm{C}^{-6}{ }^{\prime}\right), 128.8\left(\mathrm{C}-3^{\prime}, \mathrm{C}-5^{\prime}\right), 128.9(\mathrm{C}-$ $\left.4^{\prime}\right), 136.3\left(\mathrm{C}-1^{\prime}\right), 171.8(\mathrm{C}-6), 173.3(\mathrm{C}-2)$.

(3S, 5R, 1S)- and (3S, 5S, 1S)-3-sec-butyl-5phenylpiperazine-2,6-dione (3S,5S,1S)-3c and $(3 S, 5 R, 1 S)-3 c$

From (2S,1S,3S)-2c (1.81 g, $6.51 \mathrm{mmol})$ and $\mathrm{NaOH}$ (0.26 g, 1 equiv.); FC (gradient: PE/EtOAc 6:1-2:1): yield $1.44 \mathrm{~g}(90 \%): 0.75 \mathrm{~g}(47 \%)$ of $(\mathbf{3 S}, \mathbf{5 S}, \mathbf{1 S})-3 \mathrm{c}, 0.24 \mathrm{~g}$ $(15 \%)$ of $(3 S, 5 R, 1 S)-3 c$ and $0.45 \mathrm{~g}(28 \%)$ of diastereomeric mixture. $(\mathbf{3 S}, \mathbf{5 S}, \mathbf{1 S})-\mathbf{3 c}$ : white powder; mp $66-67{ }^{\circ} \mathrm{C}$; $[\alpha]_{\mathrm{D}}=-118.2\left(c 1, \mathrm{CHCl}_{3}\right)$; IR (KBr): 760, 1030, 1177, 1230, 1454, 1693, 2874, 2932, 2963, 3206; TLC (PE/AcOEt 3:1): $R_{\mathrm{f}}=0.38 ;{ }^{1} \mathrm{H}$ NMR $\left(\mathrm{CDCl}_{3}, 500 \mathrm{MHz}\right): \delta 0.94(\mathrm{t}$, $\left.{ }^{3} \mathrm{~J}=7.0,3 \mathrm{H}, \mathrm{CH}_{2} \mathrm{CH}_{3}\right), 1.07\left(\mathrm{~d},{ }^{3} \mathrm{~J}=7.0,3 \mathrm{H}, \mathrm{CH}_{3}\right), 1.26$ (m, 1H, $\left.\mathrm{CH}_{2}\right), 1.47\left(\mathrm{~m}, 1 \mathrm{H}, \mathrm{CH}_{2}^{\prime}\right), 2.20(\mathrm{~m}, 2 \mathrm{H}, \mathrm{CH}, \mathrm{NH})$, $3.30\left(\mathrm{~d},{ }^{3} J=4.5,1 \mathrm{H}, \mathrm{H}-3\right), 4.90(\mathrm{~s}, 1 \mathrm{H}, \mathrm{H}-5), 7.31-7.46(\mathrm{~m}$, $5 \mathrm{H}, \mathrm{H}-\mathrm{Ar}), 8.25$ (bs, $1 \mathrm{H}, \mathrm{CONHCO}) ;{ }^{13} \mathrm{C} \mathrm{NMR}\left(\mathrm{CDCl}_{3}\right.$, $125 \mathrm{MHz}): \delta 12.0,16.0\left(\mathrm{CH}_{3}, \mathrm{CH}_{3}^{\prime}\right), 24.6\left(\mathrm{CH}_{2}\right), 34.5(\mathrm{CH})$, 58.5 (C-3), 59.8 (C-5), $127.0\left(\mathrm{C}-2^{\prime}, \mathrm{C}-6^{\prime}\right), 128.5\left(\mathrm{C}-4^{\prime}\right)$, $129.0\left(\mathrm{C}-3^{\prime}, \mathrm{C}^{\prime} 5^{\prime}\right), 134.5\left(\mathrm{C}-1^{\prime}\right), 172.2$ (C-6), $173.2(\mathrm{C}-2)$; HRMS (ESI+) calcd for $\mathrm{C}_{14} \mathrm{H}_{18} \mathrm{~N}_{2} \mathrm{O}_{2} \mathrm{Na}$ : 269.1266 $(\mathrm{M}+\mathrm{Na})^{+}$found $269.1261 ;(\mathbf{3 S}, \mathbf{5 R}, \mathbf{1 S})-3 \mathrm{c}$ : white powder; mp 138-139 ${ }^{\circ} \mathrm{C} ;[\alpha]_{\mathrm{D}}=-94.5\left(c 1, \mathrm{CHCl}_{3}\right)$; IR $(\mathrm{KBr}): 756$, 1219, 1265, 1385, 1701, 2874, 2932, 2962, 3225; TLC (PE/ AcOEt 3:1): $R_{\mathrm{f}}=0.30 ;{ }^{1} \mathrm{H} \mathrm{NMR}\left(\mathrm{CDCl}_{3}, 500 \mathrm{MHz}\right): \delta 0.94$ $\left(\mathrm{t},{ }^{3} \mathrm{~J}=7.5,3 \mathrm{H}, \mathrm{CH}_{2} \mathrm{CH}_{3}\right), 1.08\left(\mathrm{~d},{ }^{3} \mathrm{~J}=7.0,3 \mathrm{H}, \mathrm{CH}_{3}\right)$, $1.39\left(\mathrm{~m}, 1 \mathrm{H}, \mathrm{CH}_{2}\right), 1.53\left(\mathrm{~m}, 1 \mathrm{H}, \mathrm{CH}_{2}^{\prime}\right), 1.76(\mathrm{bs}, 1 \mathrm{H}, \mathrm{NH})$, 2.29 (m, 1H, CH), 3.61 (bps, 1H, H-3), 4.52 (s, 1H, H-5), 7.36-7.42 (m, 5H, H-Ar), 8.11 (bs, $1 \mathrm{H}, \mathrm{CONHCO}) ;{ }^{13} \mathrm{C}$ NMR $\left(\mathrm{CDCl}_{3}, 125 \mathrm{MHz}\right): \delta 12.3,16.2\left(\mathrm{CH}_{3}, \mathrm{CH}_{3}^{\prime}\right), 24.7$ $\left(\mathrm{CH}_{2}\right), 35.8(\mathrm{CH}), 64.4(\mathrm{C}-3), 64.4(\mathrm{C}-5), 128.6\left(\mathrm{C}-2^{\prime}, \mathrm{C}-6^{\prime}\right)$, $128.8\left(\mathrm{C}-3^{\prime}, \mathrm{C}-5^{\prime}\right), 128.9\left(\mathrm{C}-4^{\prime}\right), 136.4\left(\mathrm{C}-1^{\prime}\right), 171.6(\mathrm{C}-6)$, 172.4 (C-2); HRMS (ESI+) calcd for $\mathrm{C}_{14} \mathrm{H}_{18} \mathrm{~N}_{2} \mathrm{O}_{2} \mathrm{Na}$ : $269.1266(\mathrm{M}+\mathrm{Na})^{+}$found 269.1280 .

(3S,5R)- and (3S,5S)-3-benzyl-5-phenylpiperazine-2,6dione (3S,5S)-3d and (3S,5R)-3d

From (2S,1S)-2d (1.02 g, $3.27 \mathrm{mmol})$ and $\mathrm{NaOH}(0.13 \mathrm{~g}$, 1 equiv.); FC (gradient: PE/EtOAc 6:1-2:1): yield $0.71 \mathrm{~g}$ (78\%): $0.44 \mathrm{~g}(48 \%)$ of (3S,5S)-3d, $0.27 \mathrm{~g}(39 \%)$ of (3S,5R)-3d. (3S,5S)-3d: white powder; mp $114-115{ }^{\circ} \mathrm{C}$; TLC (PE/AcOEt 3:1): $R_{\mathrm{f}}=0.34 ;[\alpha]_{\mathrm{D}}=-88.2 \quad(c \quad 1$, $\mathrm{CHCl}_{3}$ ); IR (KBr): 764, 1261, 1342, 1450, 1497, 1701, 2812, 3028, 3159, 3263, 3287; ${ }^{1} \mathrm{H}$ NMR $\left(\mathrm{CDCl}_{3}\right.$, $500 \mathrm{MHz}): \delta 2.12(\mathrm{bs}, 1 \mathrm{H}, \mathrm{NH}), 3.16\left(\mathrm{dd},{ }^{2} J=14.0\right.$, $\left.{ }^{3} J=8.0,1 \mathrm{H}, \mathrm{CH}_{2}\right), 3.25\left(\mathrm{dd},{ }^{2} J=14.0,{ }^{3} J=4.5,1 \mathrm{H}\right.$, 
$\left.\mathrm{CH}_{2}^{\prime}\right), 3.72\left(\mathrm{dd},{ }^{3} J_{1}=8.0,{ }^{3} J_{2}=4.5,1 \mathrm{H}, \mathrm{H}-3\right), 4.82(\mathrm{~s}$, $1 \mathrm{H}, \mathrm{H}-5), 7.21-7.36(\mathrm{~m}, 10 \mathrm{H}, \mathrm{H}-\mathrm{Ar}), 8.27$ (bs, $1 \mathrm{H}$, $\mathrm{CONHCO}) ;{ }^{13} \mathrm{C}$ NMR $\left(\mathrm{CDCl}_{3}, 125 \mathrm{MHz}\right): \delta 35.5\left(\mathrm{CH}_{2}\right)$, 54.7 (C-3), 59.8 (C-5), 127.1 (C-2', C-6'), $127.3\left(\mathrm{C}-4^{\prime \prime}\right)$, $128.5\left(\mathrm{C}-4^{\prime}\right), 128.9\left(\mathrm{C}-2^{\prime \prime}, \mathrm{C}-6^{\prime \prime}\right), 128.9\left(\mathrm{C}-3^{\prime}, \mathrm{C}-5^{\prime}\right), 129.4$ $\left(\mathrm{C}-3^{\prime \prime}, \mathrm{C}-5^{\prime \prime}\right), 134.4\left(\mathrm{C}-1^{\prime}\right), 136.3\left(\mathrm{C}-1^{\prime \prime}\right), 171.7(\mathrm{C}-6), 172.7$ (C-2); HRMS (ESI+) calcd for $\mathrm{C}_{17} \mathrm{H}_{16} \mathrm{~N}_{2} \mathrm{O}_{2} \mathrm{Na}$ : 303.1109 $(\mathrm{M}+\mathrm{Na})^{+}$found $303.1132 ;(\mathbf{3 S}, \mathbf{5 R})-\mathbf{3 d}$ : white powder; $\mathrm{mp}$ 98-99 ${ }^{\circ} \mathrm{C} ; \quad$ TLC $(\mathrm{PE} / \mathrm{AcOEt} 3: 1): \quad R_{\mathrm{f}}=0.28 ; \quad[\alpha]_{\mathrm{D}}=$ -184.2 (c 1, $\mathrm{CHCl}_{3}$ ); IR (KBr): 760, 1230, 1288, 1454, 1716, 2851, 3086, 3182; ${ }^{1} \mathrm{H}$ NMR $\left(\mathrm{CDCl}_{3}, 500 \mathrm{MHz}\right): \delta$ $1.89(\mathrm{t}, 1 \mathrm{H}, \mathrm{NH}), 2.93\left(\mathrm{dd},{ }^{2} J=14.0,{ }^{3} J=9.5,1 \mathrm{H}, \mathrm{H}-7\right)$, $3.62\left(\mathrm{dd},{ }^{2} J=14.0,{ }^{3} J=2.5, \quad 1 \mathrm{H}, \quad \mathrm{H}-7^{\prime}\right), 3.86(\mathrm{dd}$, $\left.{ }^{3} J_{1}=8.0,{ }^{3} J_{2}=2.5,1 \mathrm{H}, \mathrm{H}-3\right), 4.46(\mathrm{~s}, 1 \mathrm{H}, \mathrm{H}-5), 7.22-7.38$ (m, 10H, H-Ar), 8.18 (bs, $1 \mathrm{H}, \mathrm{NH}) ;{ }^{13} \mathrm{C} \mathrm{NMR}\left(\mathrm{CDCl}_{3}\right.$, $125 \mathrm{MHz}): \delta 36.5\left(\mathrm{CH}_{2}\right), 60.5(\mathrm{C}-3), 64.5(\mathrm{C}-5), 127.2(\mathrm{C}-$ $\left.4^{\prime \prime}\right), 128.5\left(\mathrm{C}-2^{\prime}, \mathrm{C}-6^{\prime}\right), 128.7\left(\mathrm{C}-3^{\prime}, \mathrm{C}-5^{\prime}\right), 128.8\left(\mathrm{C}-4^{\prime}\right)$, $129.0\left(\mathrm{C}-2^{\prime \prime}, \mathrm{C}-6^{\prime \prime}\right), 129.3\left(\mathrm{C}-3^{\prime \prime}, \mathrm{C}-5^{\prime \prime}\right), 136.0\left(\mathrm{C}-1^{\prime}\right), 136.7$ $\left(\mathrm{C}-1^{\prime \prime}\right), 171.4$ (C-6), 171.8 (C-2); HRMS (ESI+) calcd for $\mathrm{C}_{17} \mathrm{H}_{16} \mathrm{~N}_{2} \mathrm{O}_{2} \mathrm{Na}$ : $303.1109(\mathrm{M}+\mathrm{Na})^{+}$found 303.1115.

\section{(3S,5R)- and (3S,5S)-3,5-diphenylpiperazine-2,6-dione $(3 S, 5 S)-3 e$ and $(3 S, 5 R)-3 e$}

From diastereomeric mixture of $(2 S, 1 S)-2 \mathrm{e}$ and $(2 S, 1 R)-2 \mathrm{e}$ (1.43 g, $4.80 \mathrm{mmol})$ and $\mathrm{NaOH}(0.19 \mathrm{~g}, 1$ equiv.); $\mathrm{FC}$ (gradient: PE/EtOAc 6:1-2:1): yield $0.94 \mathrm{~g}(74 \%): 0.52 \mathrm{~g}$ $(41 \%)$ of $(3 S, 5 S)-3 e, \quad 0.42 \mathrm{~g} \quad(33 \%)$ of $(3 S, 5 R)-3 \mathrm{e}$. $(3 S, 5 S)-3 e:$ white powder; $\mathrm{mp} 126-129{ }^{\circ} \mathrm{C}$; TLC (PE/ AcOEt 3:1): $R_{\mathrm{f}}=0.17 ;[\alpha]_{\mathrm{D}}=+5.5\left(c 0.887, \mathrm{CHCl}_{3}\right) ; \mathrm{IR}$ (KBr): 700, 744, 1240, 1454, 1695, 2855, 2922, 3070, 3204, 3312; ${ }^{1} \mathrm{H}$ NMR $\left(\mathrm{CDCl}_{3}, 500 \mathrm{MHz}\right): \delta 2.48$ (bs, $1 \mathrm{H}$, $\mathrm{NH}$ ), 4.76 (s, 2H, H-3, H-5), 7.36-7.44 (m, 10H, H-Ar), 8.22 (bs, $1 \mathrm{H}, \mathrm{CONHCO}) ;{ }^{13} \mathrm{C} \mathrm{NMR}\left(\mathrm{CDCl}_{3}, 125 \mathrm{MHz}\right): \delta$ 59.5 (C-3, C-5), 127.7 (C-2', C-6', C-2" , C-6"), 128.8 (C-4', C-4"), 129.1 (C-3', C-5', C-3", C-5"), $135.2\left(\mathrm{C}-1^{\prime}, \mathrm{C}-1^{\prime \prime}\right)$, 171.5 (C-2, C-6); HRMS (ESI-) calcd for $\mathrm{C}_{16} \mathrm{H}_{13} \mathrm{~N}_{2} \mathrm{O}_{2}$ 265.0977 $(\mathrm{M}-\mathrm{H})^{-}$found 265.0982. (3S,5R)-3e: white powder; mp 172-174 ${ }^{\circ} \mathrm{C}$; TLC (PE/AcOEt 3:1): $R_{\mathrm{f}}=0.10$; $[\alpha]_{\mathrm{D}}=0\left(c 0.733, \mathrm{CHCl}_{3}\right)$; IR (KBr): 698, 737, 1219, 1263, 1454, 1709, 3034, 3065, 3103, 3223, 3317; ${ }^{1} \mathrm{H}$ NMR $\left(\mathrm{CDCl}_{3}, 500 \mathrm{MHz}\right): \delta 2.22$ (bs, $\left.1 \mathrm{H}, \mathrm{NH}\right), 4.75$ (s, 2H, H-3, $\mathrm{H}-5$ ), 7.35-7.44 (m, 6H, H-Ar), 7.45-7.49 (m, 4H, H-Ar), 8.08 (bs, $1 \mathrm{H}, \mathrm{CONHCO}) ;{ }^{13} \mathrm{C} \mathrm{NMR}\left(\mathrm{CDCl}_{3}, 125 \mathrm{MHz}\right): \delta$ 65.1 (C-3, C-5), 128.7 (C-2', C-6', C-2"', C-6"), 128.8 (C-3', C-5', C-3", C-5"), 129.0 (C-4', C-4"), $135.9\left(\mathrm{C}-1^{\prime}, \mathrm{C}-1^{\prime \prime}\right)$, 171.2 (C-2, C-6); HRMS (ESI-) calcd for $\mathrm{C}_{16} \mathrm{H}_{13} \mathrm{~N}_{2} \mathrm{O}_{2}$ $265.0977(\mathrm{M}-\mathrm{H})^{-}$found 265.0976 .

\section{(+/-)-4-Benzyl-3-phenylpiperazine-2,6-dione rac-3f}

From rac-2f $(0.32 \mathrm{~g}, 1.03 \mathrm{mmol})$ and $\mathrm{NaOH}(0.04 \mathrm{~g}$, 1 equiv.); FC (gradient: PE/EtOAc 3:1-1:1): yield $0.28 \mathrm{~g}$
(98 \%): white powder; mp $156-169{ }^{\circ} \mathrm{C}$; TLC (PE/AcOEt 3:1): $R_{\mathrm{f}}=0.22 ; \mathrm{IR}(\mathrm{KBr}): 698,744,1246,1454,1699$, 2814, 2852, 2924, 3030, 3209; ${ }^{1} \mathrm{H}$ NMR $\left(\mathrm{CDCl}_{3}\right.$, $500 \mathrm{MHz}): \delta 3.30\left(\mathrm{~d},{ }^{2} J=17.5,1 \mathrm{H}, \mathrm{PhCH}_{2}\right), 3.57(\mathrm{~d}$, $\left.{ }^{2} J=17.5,1 \mathrm{H}, \mathrm{PhCH}_{2}^{\prime}\right), 3.63\left(\mathrm{~d},{ }^{2} J=13.5,1 \mathrm{H}, \mathrm{H}-3\right), 3.83$ $\left(\mathrm{d},{ }^{2} J=13.5,1 \mathrm{H}, \mathrm{H}^{\prime}-3\right), 4.50(\mathrm{~s}, 1 \mathrm{H}, \mathrm{H}-5), 7.23-7.39(\mathrm{~m}$, 6H, H-Ar), 7.41 (m, 4H, H-Ar), 8.24 (bs, 1H, CONHCO); ${ }^{13} \mathrm{C} \mathrm{NMR}\left(\mathrm{CDCl}_{3}, 125 \mathrm{MHz}\right): \delta 51.3\left(\mathrm{PhCH}_{2}\right), 58.7(\mathrm{C}-3)$, 67.1 (C-5), 128.1, $128.8\left(\mathrm{C}-4^{\prime}, \mathrm{C}-4^{\prime \prime}\right), 128.1,128.8\left(\mathrm{C}-2^{\prime}\right.$, $\left.\mathrm{C}-6^{\prime}, \mathrm{C}-2^{\prime \prime}, \mathrm{C}-6^{\prime \prime}\right), 128.9,129.0\left(\mathrm{C}-3^{\prime}, \mathrm{C}-5^{\prime}, \mathrm{C}-3^{\prime \prime}, \mathrm{C}-5^{\prime \prime}\right)$, 134.0, $136.2\left(\mathrm{C}-1^{\prime}, \mathrm{C}-1^{\prime \prime}\right), 170.1$ (C-6), 171.0 (C-2); HRMS (ESI-) calcd for $\mathrm{C}_{17} \mathrm{H}_{15} \mathrm{~N}_{2} \mathrm{O}_{2} 279.1133(\mathrm{M}-\mathrm{H})^{-}$found 279.1126.

\section{Pharmacological evaluation}

The compounds obtained have been submitted for in vivo evaluation in the ASP of NINDS, Bethesda, USA (White et al., 2002). The experiments were performed in male albino Carworth Farms No. 1 mice (weighing 18-25 g). The animals had free access to feed and water except during the actual testing period. Housing, handling, and feeding were all in accordance with recommendations contained in the "Guide for the Care and Use of Laboratory Animals." The test compounds were dissolved or suspended in $0.5 \%(\mathrm{v} / \mathrm{v})$ aqueous solution of methylcellulose.

\section{The MES test}

In the MES test, an electrical stimulus of $0.2 \mathrm{~s}$ in duration (50 $\mathrm{mA}$ in mice) was delivered via corneal electrodes primed with an electrolyte solution containing an anesthetic agent. Mice were tested at least two different time points $(15 \mathrm{~min}, 30 \mathrm{~min}, 1 \mathrm{~h}$ or $4 \mathrm{~h}$ ) following intraperitoneal administration of 30,100 , and $300 \mathrm{mg} / \mathrm{kg}$ of test compound. Abolition of the hindlimb tonic extensor component indicated the test compound's ability to inhibit MES-induced seizure spread (White et al., 2002).

\section{The scMET seizure test}

The test utilized a dose of metrazole (pentylenetetrazole, $85 \mathrm{mg} / \mathrm{kg}$ in mice). This produced clonic seizures lasting for a period of at least $5 \mathrm{~s}$ in $97 \%\left(\mathrm{CD}_{97}\right)$ of animals tested. At the anticipated time of testing, the convulsant was administered subcutaneously. The test compound was administered intraperitoneally in mice and the animals were observed over a $30 \mathrm{~min}$ period. Mice were tested at least two different time points $(15 \mathrm{~min}, 30 \mathrm{~min}, 1 \mathrm{~h}$ or $4 \mathrm{~h}$ ) following intraperitoneal administration of 30,100 , and $300 \mathrm{mg} / \mathrm{kg}$ of test compound. The absence of clonic spasms indicated a compound's ability to abolish the effect of pentylenetetrazol on seizure threshold (White et al., 2002). 
The acute neurological impairment (TOX)

Neurological toxicity induced by a compound was detected in mice or rats using the standardized rotorod test (Dunham and Miya, 1957). Mice were tested at a minimum of two different time points $(15 \mathrm{~min}, 30 \mathrm{~min}, 1 \mathrm{~h}$ or $4 \mathrm{~h}$ ) following intraperitoneal administration of $100 \mathrm{mg} / \mathrm{kg}$ of test compound. Rats were tested at time intervals between 0.25 and $4 \mathrm{~h}$ following an oral or intraperitoneal dose of $30 \mathrm{mg} /$ $\mathrm{kg}$. Neurological impairment was demonstrated by the inability of animals to maintain equilibrium on a $6 \mathrm{rpm}$ rotation rod for a given time.

The minimal clonic seizure test $(6 \mathrm{~Hz})$

The $6 \mathrm{~Hz}$ screen was carried out according to the protocol originally described by Brown et al. (1953) and more recently by Barton et al. (2001) and Kaminski et al. (2004). It is an alternative electroshock paradigm that uses lowfrequency $(6 \mathrm{~Hz})$, long-duration (3 s) electrical stimulation. Mice were tested at time intervals between 0.25 and $4 \mathrm{~h}$ following intraperitoneal doses of $100 \mathrm{mg} / \mathrm{kg}$ of test compound. Corneal stimulation $(0.2 \mathrm{~ms}$-duration monopolar rectangular pulses at $6-\mathrm{Hz}$ for $3 \mathrm{~s}$ ) was delivered by a constant-current device. During the stimulation, mice were manually restrained and released into the observation cage immediately after the current application. The seizures manifested in "stunned" posture associated with rearing, forelimb, automatic movements and clonus, twitching of the vibrissae and Straub-tail. The duration of the seizure activity ranged from 60 to $120 \mathrm{~s}$ in untreated animals. At the end of the seizure, animals resumed their normal exploratory behavior. The experimental end point was protection against the seizure. The animal was considered to be protected if it resumed its normal exploratory behavior within $10 \mathrm{~s}$ from the stimulation (Kaminski et al., 2004).

Acknowledgments We would like to thank the Anticonvulsant Screening Program (National Institute of Neurological Disorders and Stroke, NINDS Rockville, MD, USA) staff for providing the pharmacological data. We would also like to thank Carolyn Foster for her assistance in preparation of the manuscript. Financial support was provided by the Polish Ministry of Science and Higher Education (Grant No. N N405 623138).

Open Access This article is distributed under the terms of the Creative Commons Attribution License which permits any use, distribution, and reproduction in any medium, provided the original author(s) and the source are credited.

\section{References}

Banerjee PS, Sharma PK (2012) New antiepileptic agents: structureactivity relationships. Med Chem Res 21:1491-1508
Barton ME, Klein BD, Wolf HH, White HS (2001) Pharmacological characterization of the $6 \mathrm{~Hz}$ psychomotor seizure model of partial epilepsy. Epilepsy Res 47:217-227

Bialer M, White HS (2010) Key factors in the discovery and development of new antiepileptic drugs. Nat Rev Drug Discov 9:68-82

Bialer M, Johannessen SI, Levy RH, Perucca E, Tomson T, White HS (2013) Progress report on new antiepileptic drugs: a summary of the Eleventh Eilat Conference (EILAT XI). Epilepsy Res 103:2-30

Brodie MJ (2001) Do we need any more antiepileptic drugs? Epilepsy Res 45:3-6

Brown WC, Schiffman DO, Swinyard EA, Goodman LS (1953) Comparative assay of an antiepileptic drugs by psychomotor seizure test and minimal electroshock threshold test. J Pharmacol Exp Ther 107:273-283

Dawidowski M, Herold F, Chodkowski A, Kleps J, Szulczyk P, Wilczek M (2011) Synthesis and anticonvulsant activity of novel 2,6-diketopiperazine derivatives. Part 1: perhydropyrrole[1,2a]pyrazines. Eur J Med Chem 46:4859-4869

Dawidowski M, Herold F, Chodkowski A, Kleps J (2012a) Synthesis and anticonvulsant activity of novel 2,6-diketopiperazine derivatives. Part 2: perhydropyrido[1,2-a]pyrazines. Eur J Med Chem 48:347-353

Dawidowski M, Herold F, Turło J, Wilczek M, Chodkowski A, Gomółka A, Kleps J (2012b) Synthesis of bicyclic 2,6-diketopiperazines via a three-step sequence involving an Ugi fivecenter, four-component reaction. Tetrahedron 68:8222-8230

Demharter A, Hörl W, Eberhardt H, Ugi I (1996) Synthesis of chiral $1,1^{\prime}$-iminodicarboxylic acid derivatives from $\alpha$-amino acids, aldehydes, isocyanides, and alcohols by the diastereoselective five-center-four-component reaction. Angew Chem Int Ed 35:173-175

Dunham MS, Miya TA (1957) A note on a simple apparatus for detecting neurological deficit in rats and mice. J Am Pharm Assoc Sci Ed 46:208-209

Kaminski RF, Livingood MR, Rogawski MA (2004) Allopregnanolone analogs that positively modulate GABA receptors protect against partial seizures induced by $6-\mathrm{Hz}$ electrical stimulation in mice. Epilepsia 45:864-867

Kwan P, Brodie MJ (2000) Early identification of refractory epilepsy. N Engl J Med 342:314-319

Löscher W, Schmidt D (1994) Strategies in antiepileptic drug development: is rational drug design superior to random screening and structural variation? Epilepsy Res 17:95-134

Malawska B (2005) New anticonvulsant agents. Curr Top Med Chem 5:69-85

Mishra R, Ganguly S (2012) Imidazole as an anti-epileptic: an overview. Epilepsy Res 21:3929-3939

Perucca E, French J, Bialer M (2007) Development of new antiepileptic drugs: challenges, incentives, and recent advances. Lancet Neurol 6:793-804

Rogawski MA (2006) Diverse mechanisms of antiepileptic drugs in the development pipeline. Epilepsy Res 69:273-294

Smith M, Wilcox KS, White HS (2007) Discovery of antiepileptic drugs. Neurotherapeutics 4:12-17

White HS, Woodhead JH, Wilcox KS, Stables JP, Kupferberg HJ, Wolf HH (2002) General principles: discovery and preclinical development of antiepileptic drugs. In: Levy RH, Mattson RH, Meldrum BS, Perucca E (eds) Antiepileptic drugs, 5th edn. Lippincott Williams and Wilkins Publishers, New York, pp 6-48 